\title{
Multiobjective Auction-Based Switching-Off Scheme in Heterogeneous Networks: To Bid or Not to Bid?
}

\author{
Alexandra Bousia, Member, IEEE, Elli Kartsakli, Senior Member, IEEE, \\ Angelos Antonopoulos, Senior Member, IEEE, Luis Alonso, Senior Member, IEEE, and \\ Christos Verikoukis, Senior Member, IEEE
}

6 Abstract-The emerging data traffic demand has caused a mas7 sive deployment of network infrastructure, including macro base 8 stations (BSs) and small cells (SCs), leading to increased energy 9 consumption and expenditures. However, the network underuti10 lization during low traffic periods (e.g., night zone) enables the 11 mobile network operators (MNOs) to save energy by having their 12 traffic served by third-party SCs, thus being able to switch off 13 their BSs. In this paper, we propose a novel market approach to 14 foster the opportunistic utilization of the unexploited SCs capacity, 15 where the MNOs, instead of requesting the maximum capacity 16 to meet their highest traffic expectations, offer a set of bids re17 questing different amounts of resources from the third-party SCs 18 at lower costs. Motivated by the conflicting financial interests of 19 the MNOs and the third party, the restricted capacity of the SCs 20 that is not adequate to carry the whole traffic in multioperator 21 scenarios, and the necessity for energy-efficient solutions, we in22 troduce a combinatorial auction framework, which includes 1) a 23 bidding strategy, 2) a resource-allocation scheme, and 3) a pricing 24 rule. We propose a multiobjective framework as an energy- and 25 cost-efficient solution for the resource-allocation problem, and we 26 provide extensive analytical and experimental results to estimate 27 the potential energy and cost savings that can be achieved. In 28 addition, we investigate the conditions under which the MNOs 29 and the third-party companies should take part in the proposed 30 auction.

31 Index Terms-Auction, energy efficiency, game theory, green 32 networking, heterogeneous networks (HetNets), multiobjective op33 timization, offloading, switching off.

\section{A. Motivation}

36 The rapid expansion of mobile services, along with the 37 emerging demand for multimedia applications, driven by the 38 widespread use of laptops, tablets, and smart devices, has led

Manuscript received April 22, 2015; revised August 7, 2015, October 5, 2015, and December 24, 2015; accepted December 30, 2015. This work was supported in part by the Research Project AGAUR under Grant 2014-SGR1551, by the Research Project CellFive under Grant TEC2014-60130-P, and by the Research Project DEFINE-5G under Grant TEC2014-60258-C2-2-R. The review of this paper was coordinated by Prof. W. Song.

A. Bousia, E. Kartsakli, and L. Alonso are with the Department of Signal Theory and Communications, Universitat Politècnica de Catalunya, 08034 Barcelona, Spain (e-mail: alexandra.bousia@tsc.upc.edu; ellik@tsc.upc.edu; luisg@tsc.upc.edu).

A. Antonopoulos and C. Verikoukis are with Telecommunications Technological Center of Catalonia, 08860 Barcelona, Spain (e-mail: aantonopoulos@ cttc.es; cveri@cttc.es).

Color versions of one or more of the figures in this paper are available online at http://ieeexplore.ieee.org.

Digital Object Identifier 10.1109/TVT.2016.2517698 to an impressive growth of data traffic during the last few 39 years. Global mobile data traffic is expected to increase nearly 40 tenfold, reaching 24.3 EB per month by 2019 [1]. Hence, 41 mobile network operators (MNOs ${ }^{1}$ ) seek to extend their in- 42 frastructure by installing more base stations (BSs). In an effort 43 to increase the capacity of their network and meet these press- 44 ing traffic demands, they also lease capacity and bandwidth 45 resources from third parties, who deploy low-powered small- 46 cell (SC) networks to provide enhanced services via traffic 47 offloading from macro BSs to SCs during peak hours [2]. For 48 instance, Nokia Networks [3] has recently built networks of 49 interconnected SCs, enabling operators to extend the coverage 50 and increase the capacity of existing macro networks. The 51 involvement of various entities of corporate nature with differ- 52 ent financial goals generates a new ecosystem with interesting 53 dynamics to be studied. To that end, in [4], the economics 54 of offloading are investigated, whereas auction theory is used 55 to model economic transactions between conflicting parties in 56 several works [5]-[7] through offloading schemes with auction- 57 based resource-allocation problems.

The dense heterogeneous networks (HetNets) imply sig- 59 nificant increase in the capital and operational expenditures 60 (CapEx and OpEx) of MNOs [8], and as a result, there is a 61 strong motivation to investigate energy-efficient solutions to 62 bring down the energy consumption and the cost of networks. 63 This goal can be accomplished through the switching off of the 64 underutilized nodes when the traffic is significantly low. Since 65 the BSs are the most power hungry and expensive components 66 of the networks [9], the research community has shifted toward 67 the investigation of BS switching-off schemes [10]-[15]. De- 68 spite their promising results, these works examine only one tier 69 of macro BSs, whereas the presence of multiple MNOs raises 70 new challenges and open issues.

The SC deployment in current HetNets can be the key to 72 implementing novel energy and cost-efficient solutions. By 73 encouraging the traffic offloading from BSs to SCs during low 74 traffic periods, when the SC resources are most likely to remain 75 unused, part of the BS infrastructure can be switched off. Given 76 that the energy consumption of the BSs is considerably higher 77 with respect to the SCs, even when the traffic load is low, such 78 solutions can yield high energy gains for the MNOs. Thus, 79 offloading can be exploited to concentrate the users to the SCs 80

\footnotetext{
${ }^{1}$ The terms "MNO" and "operator" will be used interchangeably in the remainder of this paper.
} 
81 and allow the BSs with no traffic to be switched off. However, 82 despite the potential gains of the opportunistic exploitation of 83 the capacity of SCs and the deactivation of the redundant BSs 84 during low traffic conditions, very few research works exist in 85 the literature so far.

86 An overview of the existing techniques in energy-efficient 87 network planning is presented in [16], highlighting the advan88 tages and the shortcomings of the algorithms and revealing 89 the open issues that should be further studied in the field of 90 HetNets and operators' collaboration. In the same context, in 91 [17], an auction-based offloading scheme is proposed for the BS 92 switching off, where the operators submit a bidding value, and a 93 third party's income optimization approach is followed to solve 94 the resource-allocation problem. However, only a particular 95 network configuration is considered, where it is assumed that 96 the SC capacity is sufficient to serve the traffic of the network, 97 allowing the switching off of all the BSs.

98 Despite the clear benefits of the BSs switching off via of99 floading, the presence of multiple MNOs in the same area [18] 100 would complicate the application of this scheme since the SCs 101 may not be able to fully support the network traffic. Another 102 limitation of the auction-based state-of-the-art works [5], [6], 103 [17] consists in the fact that the MNOs propose a single 104 bidding value for the requested capacity, based on the maximum 105 predictions about the traffic that they are willing to offload. 106 However, these predictions do not always correspond to the 107 real traffic values, which can be significantly lower than the 108 maximum values, leading to increased costs for the operators 109 and inefficient use of the SC capacity resources.

\section{B. Contribution}

111 In this paper, motivated by the aforementioned issues, we 112 propose a novel energy-efficient solution for dense HetNets, 113 which considers the interests of the involved parties (MNOs and 114 third party) and the time-varying traffic characteristics. More 115 specifically, we introduce an offloading mechanism, where the 116 operators lease the capacity of an SC network owned by a third 117 party, to be able to switch off their BSs and maximize their 118 energy efficiency, when the traffic demand is low. The MNOs 119 request capacity from several SCs and can only switch off their 120 BSs if all their requests are satisfied, enabling them to offload 121 all their traffic to the SC network.

122 To that end, the allocation of the SC resources among a 123 set of competing MNOs is mathematically formulated as an 124 auction. Since the exact resource requirements of the network 125 are unknown beforehand, the MNOs employ past reports to 126 predict the maximum expected traffic load. Then, exploiting 127 the fact that, with a high probability, the actual traffic will 128 be lower than the predicted maximum (especially during low 129 traffic periods), the MNOs submit a set of bids to the SCs, 130 requesting for lower capacity resources (with respect to the 131 maximum estimated requirements). With this approach, the SC 132 resources can be more efficiently utilized, and the MNOs are 133 likely to pay a lower price for the leased capacity, taking a 134 small risk of not being able to serve all users under some 135 circumstances (i.e., when the traffic approaches the maximum 136 predictions and the leased capacity is not sufficient). Our key contribution is to study this very interesting trade-off between 137 the potential energy and financial gains of this solution and 138 the risk of not fully satisfying all the users, thus providing the 139 MNOs with the necessary insights to decide whether it is prof- 140 itable to participate in the auction, depending on their tolerance 141 to the potential loss of some users.

In addition, the conflicting interests of the involved parties 143 are also taken into consideration. On the one hand, the MNOs 144 aim to reduce their energy consumption and expenditures by 145 offloading their traffic and switching off their BS infrastructure. 146 However, to deactivate a BS, all its traffic should be offloaded 147 to the SC network (i.e., the MNO should win in all the auctions 148 involving the particular BS). On the other hand, the third 149 party wants to maximize its income by leasing the maximum 150 possible amount for resources to the MNOs. However, the 151 resource allocation policy that maximizes the third party's 152 income may not enable the operators to switch off their BSs. 153 Our proposed auction-based strategy takes into account these 154 conflicting interests to achieve a feasible, efficient, and energy- 155 saving resource-allocation scheme.

In summary, the contribution of this paper is described as 157 follows.

1) Bidding Strategy: We propose a novel bidding strategy, 160 where MNOs submit a set of bids (and not only one bid) 161 to the third party, requesting different capacity resources, 162 based on the predictions about their maximum traffic. The 163 diversity of bids allows more offloading opportunities, 164 which is profitable for both the MNOs and the third party. 165

2) Auction Design and Switching Off Decision: We design 166 an auction scheme that enables the efficient usage of SCs 167 resources under low traffic conditions. Our framework 168 motivates the MNOs to quantify their tolerance about 169 requesting fewer resources and form the different levels 170 of bids. We show that the proposed auction-based scheme 171 has two desirable properties: 1) truthfulness; and 2) indi- 172 vidual rationality. The mechanism is designed based on a 173 multiobjective framework, where the conflicting interests 174 of the involved parties are considered, to provide the 175 optimal solution that maximizes the economic profit of 176 both the third party and the MNOs and minimizes the 177 network energy consumption at the same time.

3) Performance Evaluation: We validate the theoretical 179 analysis of the multiobjective problem by computing 180 the Pareto front (i.e., the set of optimal) solutions and 181 assess the effectiveness of the proposed auction-based 182 switching-off algorithm. The analytical and simula- 183 tion results indicate the potential energy efficiency and 184 economic gains in the network and give the necessary 185 insights to the MNOs to decide whether it is beneficial 186 to enter in a resource allocation negotiation with the 187 third party.

The remainder of this paper is organized as follows. The 189 system model is described in Section II. In Section III, we in- 190 troduce the auction-based optimization approach that is used for 191 the BSs' switching-off decision. The performance evaluation is 192 provided in Sections IV and V concludes this paper. 


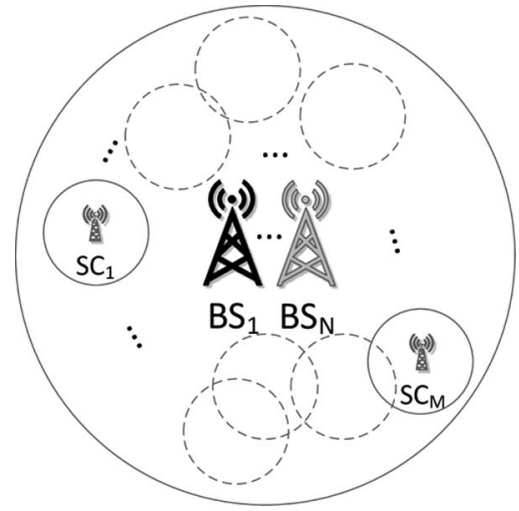

Fig. 1. Network configuration with one macro cell served by $N$ MNOs and covered by $M$ third-party SCs.

\section{A. Network Configuration}

196 We consider an urban scenario, focusing on an area of a 197 macro cell. In the macro cell, we assume that $N$ MNOs provide 198 coverage through their BSs, which are denoted by $\mathrm{BS}_{n}$, where $199 n \in \mathcal{N}=\{1, \ldots, N\}$ characterizes the $\mathrm{MNO}_{n}$. Let us high200 light that, in dense networks, due to legal regulations, MNOs 201 are obligated to install their antennas and BSs on the same 202 buildings; thus, we examine networks with collocated BSs [18]. 203 Moreover, in the macro cell, SCs, owned by a third party, are 204 randomly distributed. Each $\mathrm{SC}$ is represented as $\mathbf{S C}_{m}$, where $205 m \in \mathcal{M}=\{1, \ldots, M\}$. We assume that the number of SCs is 206 adequate to cover the area of the macro cell [19]. As it will be 207 explained in the following, the MNOs are motivated to offload 208 their traffic to the third-party SCs by paying the corresponding 209 price, thus enabling part of BS infrastructure to be switched 210 off during low traffic conditions. The network configuration is 211 shown in Fig. 1.

\section{B. Traffic Load Model}

213 In this paper, we adopt a realistic traffic pattern [20], [21] 214 that corresponds to the maximum traffic per operator in a given 215 cell. Fig. 2(a) plots the maximum traffic per hour, which is 216 denoted by Load $^{\max }(h)$, throughout the day. ${ }^{2}$ Without loss of 217 generality, we focus on the time zone between 01:00 A.M. and 218 09:00 A.M., when the traffic per BS is relatively low (i.e., less 219 than $40 \mathrm{Mb} / \mathrm{s}$, which corresponds to $35 \%$ of the cell's capacity). 220 The selection of the night zone may vary according to the traffic 221 variations, and our algorithm can be adapted to different traffic 222 conditions. In addition, we assume that the traffic volumes of 223 different MNOs may be different, although they follow the 224 same pattern. Hence, we define $\rho_{n} \in[0,1]$ as the percentage of 225 each operator's traffic load with respect to the maximum traffic 226 for the respective hour. In this paper, we have used traffic data 227 sets that include information of one operator for ten BSs during 228 the period of one year, thus including weekends, weekdays, 229 and day and night hours. To that end, the reports consists of 230 various values for both low and high traffic. Provided that the

\footnotetext{
${ }^{2}$ The parameter $h$ can be dropped for the sake of simplicity.
}

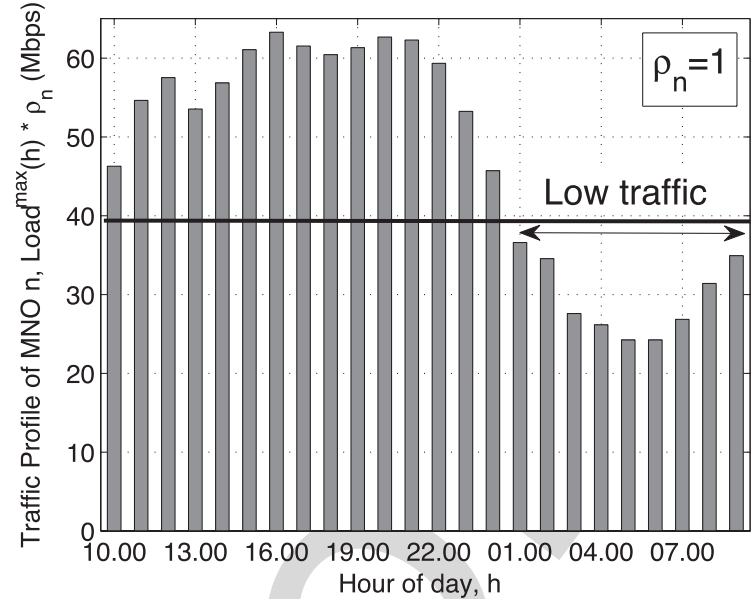

(a)

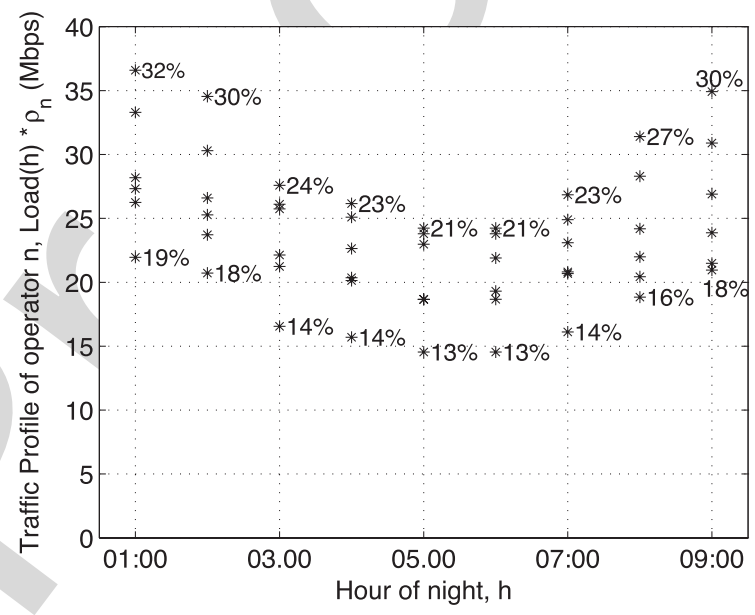

(b)

Fig. 2. Traffic pattern scenario and real data traffic values. (a) Traffic load during the 24-hour day. (b) Real data for traffic load patterns.

data sets had huge information, we classified the days of the 231 year into different periods of time (e.g., seasons, weekdays, 232 and weekends). Based on these real traffic reports and by 233 using simple statistical analysis, we have obtained the necessary 234 insights about the minimum, maximum, and average values of 235 the traffic load, and we have plotted some indicative numbers in 236 Fig. 2(b). Each one of the six values corresponds to the average 237 traffic that was observed in different days during the year. In 238 addition, we express the BS utilization as a percentage of the 239 total BS's capacity resources for the extreme cases of minimum 240 and maximum traffic loads. The two values, i.e., Load ${ }^{\min }$ and 241 Load $^{\text {max }}$, are very critical, and it is very important for the 242 MNOs to be able to predict them.

Unlike the existing works in the literature, where each MNO 244 places only one bid corresponding to the maximum capacity 245 requirements, in this paper, we propose that the MNOs place 246 multiple bids corresponding to different levels of the predicted 247 traffic load, as shown in Fig. 2(b). Thus, given the estimated 248 minimum and maximum traffic load levels and assuming that 249 the actual traffic values will, most probably, lie between these 250 extreme values, we are able to calculate different levels of 251 traffic. In this paper, we consider $L+1$ different traffic load 252 levels. The number of levels depends on the MNOs' strategy 253 


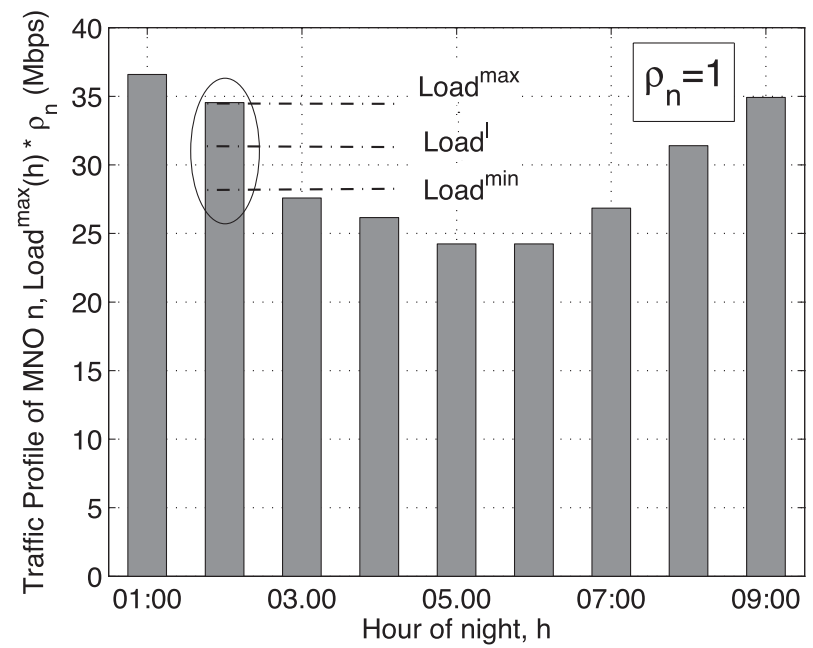

Fig. 3. Traffic during the night zone and different levels of traffic estimation.

254 that will be further explained in the following. Each level $l \in$ $255 \mathcal{L}=\{0, \ldots, L\}$ corresponds to traffic that is equal to

$$
\begin{aligned}
\operatorname{Load}^{l} & =\operatorname{Load}^{\text {min }}+\frac{\operatorname{Load}^{\text {max }}-\operatorname{Load}^{\text {min }}}{L} \cdot l \\
& =\left(1-\frac{l}{L}\right) \cdot \operatorname{Load}^{\text {min }}+\frac{l}{L} \cdot \operatorname{Load}^{\text {max }} .
\end{aligned}
$$

256 Evidently, the two extreme values are $\operatorname{Load}^{0}=\operatorname{Load}^{\mathrm{min}}$ and 257 Load $^{L}=$ Load $^{\text {max }}$. Fig. 3 shows an example, depicting various 258 traffic levels for a specific hour $(\mathrm{h}=02: 00$ A.M.). Based 259 on the above, Appendix A of the supplemental file provides 260 a theoretical estimation of the traffic that can be offloaded, 261 explaining the process of mapping the users of the MNOs to 262 the capacity of the SCs.

\section{III. Auction-Based Switching-OfF Algorithm}

264 Here, we present the combinatorial auction employed to 265 select the MNOs that can offload their traffic to the SCs, thus 266 being able to switch off their BSs. We provide the bidding strat$267 \mathrm{egy}$, and we formulate the integer linear programming model, 268 which ensures the optimal allocation and the switching-off 269 strategy for the auction. In addition, to ensure truthful bidding, 270 the Vickrey-Clarke-Grooves (VCG) payment mechanism 271 [22] is employed.

\section{A. Big Picture}

273 By considering the limited capacity of the SCs and the 274 MNOs' incentive to switch off their BSs, we use an auction275 based mechanism to motivate the operators to offload the traffic 276 to the third-party SC network. Fig. 4 illustrates the main idea of 277 the scheme. In our proposal, both the MNOs and the third party 278 take part in the decision process, each one having a separate 279 role in the framework. On one hand, the MNOs act as buyers, 280 who are willing to lease the SCs resources and opportunistically 281 offload their traffic. On the other hand, the third party, acting as 282 a seller, collects the bids, and through an auction, the subset of 283 MNOs that can offload their traffic, is selected. The proposed 284 auction-based switching-off scheme consists of three main
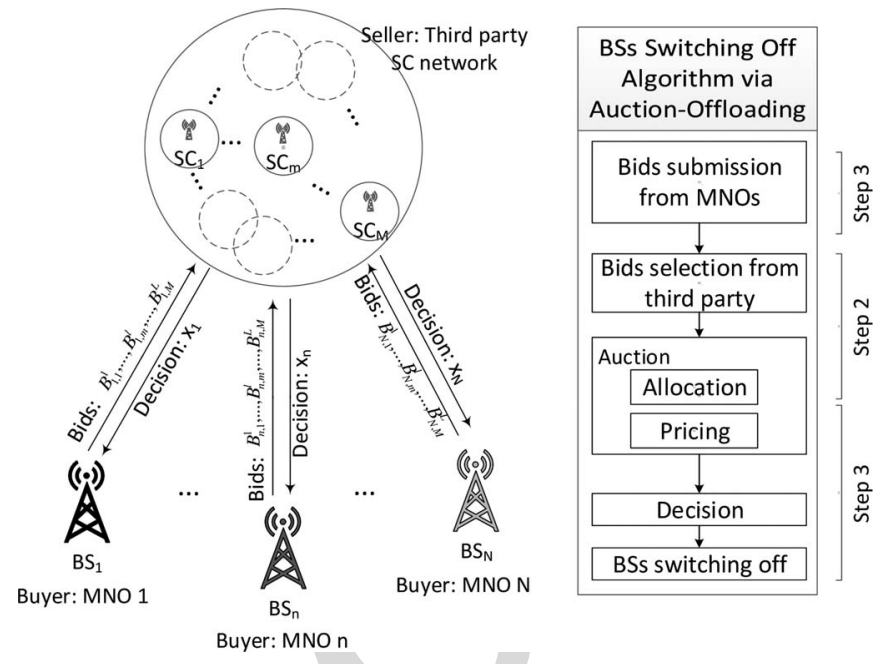

Fig. 4. Auction illustration and proposed algorithm flowchart.

steps: bidding, allocation, and pricing, whose process and the 285 respective decision-makers (in parenthesis) are presented as fol- 286 lows. First, in the bidding phase (MNOs), the MNOs place their 287 bids to the third party according to the different values of the 288 requested bandwidth. Each bid includes the information of the 289 requested capacity and the corresponding price that the MNO is 290 willing to offer. Second, in the allocation step (third party and 291 MNOs), the third party collects the MNOs' bids. The selection 292 of the optimal resource allocation can be derived through the so- 293 lution of the resource-allocation problem. Third, in the pricing 294 step (third party), the third party decides each winner's payment 295 price, based on the resource allocation of the previous step. The 296 winning MNOs offload their whole traffic to the SCs and switch 297 off their BSs, whereas the losing bidders keep their BSs active. 298

\section{B. Bidding Strategy}

Each $\mathrm{SC}_{m}$ of the third party (seller) has unexploited capacity 300 resources that is willing to lease to the MNOs. The MNOs 301 (buyers) want to offload their traffic to the SCs by requesting 302 specific capacity resources to lease, based on the predictions 303 of the traffic load. The number of the physical resource blocks 304 (PRBs) is calculated in Appendix A of the supplementary 305 file. The MNOs valuate the requested resource $N_{\mathrm{PRB}_{n, m}}^{l}$ at a 306 given price $u_{n, m}^{l}$, unknown to the third party and the other 307 bidders, where $l$ is the level of resources, $n \in \mathcal{N}$ refers to 308 the corresponding operator, and $m \in \mathcal{M}$ corresponds to the 309 specific SC that the MNO wants to lease the resources from. In 310 the proposed auction-based scheme, each operator submits a set 311 of bid pairs $B_{n, m}^{l}=\left(b_{n, m}^{l}, N_{\mathrm{PRB}_{n, m}}^{l}\right)$, representing the price 312 $b_{n, m}^{l} \leq u_{n, m}^{l}$, which the $n$th MNO pays for leasing the capacity 313 $N_{\mathrm{PRB}_{n, m}}^{l}$ from the $m$ th SC. In general, these two values (i.e., 314 $b_{n, m}^{l}, u_{n, m}^{l}$ ) may not necessarily be the same. However, in a 315 truthful auction such as the one that we have (the truthfulness 316 property of the auction will be explained in Section III-D), it 317 is proved that the private valuation and the bidding price are 318 equal; thus, $b_{n, m}^{l}=u_{n, m}^{l}$ [5], [6]. After the reception bid pairs, 319 the selection of the subset of the MNOs, whose traffic can be 320 offloaded to the corresponding SCs, follows. 


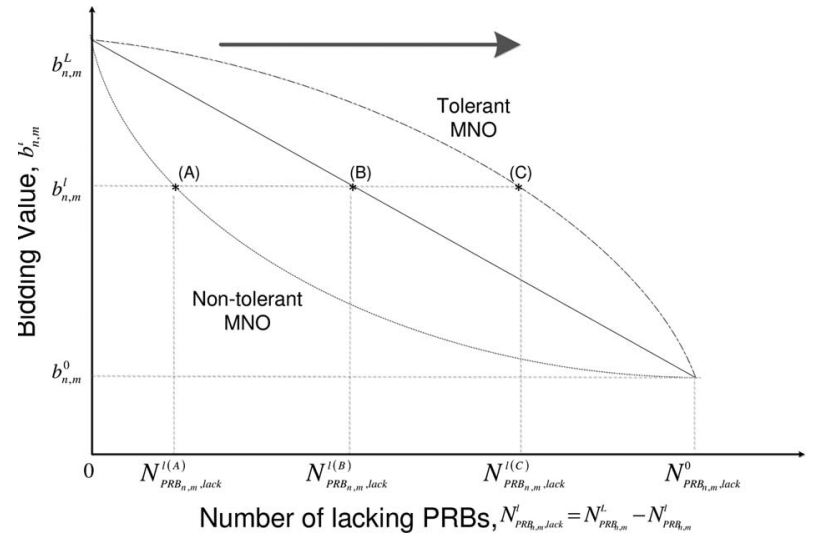

Fig. 5. Bidding strategy versus tolerance function.

322 To gain further insights on the bidding strategy, let us 323 consider the following example. Given the predicted expecta324 tions about the traffic, each MNO $m$ submits multiple bids, $325 b_{n, m}^{0}, b_{n, m}^{1}, \ldots, b_{n, m}^{l}, \ldots, b_{n, m}^{L}$, indicating the offered price for 326 the requested number of PRBs. Through the different bid pairs, 327 the MNOs actually reveal their outage probability tolerance. 328 In particular, by leasing the maximum calculated number of 329 PRBs $\left(N_{\mathrm{PRB}_{n, m}}^{L}\right)$ for the highest bid $b_{n, m}^{L}$, the MNOs guarantee 330 service to all their users. Controversially, by obtaining a smaller 331 number of PRBs (e.g., $N_{\mathrm{PRB}_{n, m}}^{l}$ with $l<L$ ), the MNO pays 332 a smaller price at a risk of leaving some users in outage. 333 The minimum number of requested PRBs $N_{\mathrm{PRB}_{n, m}}^{0}$ constitutes 334 an upper bound of the operator's outage tolerance. Another 335 parameter determined by each MNO is the number of levels. 336 A higher number of levels results in more bid pairs, thus 337 increasing the performance of the auction while inducing more 338 communication overhead and higher computational complexity. 339 To flexibly model the MNOs' outage tolerance, we introduce 340 a satisfaction function that represents the bidding value (which 341 is equal to the valuation price) that the MNO is willing to 342 pay for leasing a specific bandwidth. To that end, we define 343 the number of lacking PRBs as the difference between the 344 maximum number of PRBs minus the actually obtained PRBs 345 allocated the MNO, given by

$$
N_{\mathrm{PRB}_{n, m}, \text { lack }}^{l}=N_{\mathrm{PRB}_{n, m}}^{L}-N_{\mathrm{PRB}_{n, m}}^{l} .
$$

346 The satisfaction function is determined by the requested ca347 pacity of each MNO and is monotonically decreasing with the 348 number of lacking PRBs. We also consider that, for each MNO, 349 there is a lower bound for the minimum number of requested 350 PRBs, which minimizes the MNO's satisfaction. This bound 351 indicates that for fewer PRBs the MNO will not participate in 352 the auction. Fig. 5 shows three examples of the outage tolerance 353 function. Three bidding values are emphasized in the plot: The 354 highest bid $b_{n, m}^{L}$, corresponding to the maximum requested 355 capacity $N_{\mathrm{PRB}_{n, m}}^{L}$ (with $N_{\mathrm{PRB}_{n, m} \text {, lack }}^{l}=0$ ), the lowest bid $356 b_{n, m}^{0}$, and an intermediate value $b_{n, m}^{l}$. As the number of lacking 357 PRBs increases (depicted by the arrow direction in Fig. 5), the 358 bid values decrease, and as a consequence, losses in terms of the 359 served users may be observed. If the number of lacking PRBs 360 exceeds $N_{\mathrm{PRB}_{n, m}}^{0}$, lack , the MNO will not participate in the auc- tion; thus, no bid lower than $b_{n, m}^{0}$ will be submitted. The three 361 points (A, B, and C) marked in Fig. 5 correspond to the different 362 tolerance functions of three operators. An outage-tolerant MNO 363 (point $\mathrm{C}$ ) requests for less bandwidth for the same bidding value 364 $b_{n, m}^{l}$ compared with a nontolerant MNO (point A) that still 365 requests high capacity from the third party. Hence, the nontol- 366 erant MNO places higher priority on guaranteeing user service, 367 whereas the outage-tolerant MNO is willing to sacrifice some 368 resources to increase its probability of winning the auction and 369 switching off its BS, thus enhancing energy efficiency. Finally, 370 point B corresponds to an intermediate bidding strategy defined 371 by a linear function between the two examined parameters. 372

The user outage tolerance function (keeping in mind that 373 $\left.b_{n, m}^{l}=u_{n, m}^{l}\right)$ is modeled as ${ }^{3}$

$$
b_{n, m}^{l}=b_{n, m}^{L}-\frac{b_{n, m}^{L}-b_{n, m}^{0}}{N_{\mathrm{PRB}_{n, m}}^{L}-N_{\mathrm{PRB}_{n, m}}^{0}} \cdot\left(N_{\mathrm{PRB}_{n, m}}^{l}\right)^{\delta} .
$$

The distinctive cases for the satisfaction curves are as 375 follows.

- Nontolerant MNO (Point A): For the nontolerant MNO, 378 the outage tolerance function is convex, with $\delta<1$. 379

- Average-tolerant MNO (Point B). There is a linear re- 380 lation between the bidding strategies and the outage 381 tolerance of the MNOs, by substituting $\delta=1$ in (3). $\quad 382$

- Tolerant MNO (Point C). For the tolerant MNO, the 383 satisfaction function is concave, with $\delta>1$.

The parameter $\delta$ may obtain a wide range of values. The selec- 385 tion of a value equal to $\delta \ll 1$ corresponds to a strictly nontoler- 386 ant MNO, who decreases its bid requests very fast. In contrast, a 387 very high value of this indicative parameter (i.e., $\delta \gg 1$ ) would 388 lead to very slowly decreasing bidding values and, thus, to a 389 very tolerant operator.

\section{Auction Formulation}

By employing the properties of combinatorial auction theory, 392 we formulate the problem of the opportunistic offloading as 393 an auction. Each MNO $n \in \mathcal{N}$ places the corresponding bid 394 pairs $B_{n, m}^{l}$. Having received the bids, the third party selects 395 the subset of MNOs that maximizes the desired goals of all the 396 involved participants. We define $x_{n, m}^{l}$ as a binary decision vari- 397 able that indicates whether the corresponding bidder (MNO $n$ ) 398 is winner $\left(x_{n, m}^{l}=1\right)$ or not $\left(x_{n, m}^{l}=0\right)$ in the corresponding 399 $\mathrm{SC}_{m}$ and for the $l$ th bidding value.

400

The cost of using the SC infrastructure and the increased 401 consumed energy are the main objectives: the maximization 402 of the profits (the economic objectives of the third party and 403 the MNOs) and the minimization of the energy consumption 404 (the energy objective). In continuation, a detailed analysis of 405 the objectives of each party is presented.

406

1) Third Party's Objective: The third party aims at maximiz- 407 ing its profit by leasing high volumes of the capacity of SCs at 408

\footnotetext{
${ }^{3}$ The values of $b_{n, m}^{0}$ and $b_{n, m}^{L}$ will be calculated in details in the succeeding section, where the constraints and requirements posed by the MNOs and the third party are given within the optimization framework.
} 
409 increased prices. The profit is defined as the difference of the 410 MNOs' winning bids minus its expenses (CapEx and OpEx). 411 We define the financial gain of the $\mathrm{SC}$ network $\mathrm{CG}_{\mathrm{SC}}$ as

$$
f_{1}(\mathbf{x})=\mathrm{CG}_{\mathrm{SC}}=\sum_{n \in \mathcal{N}} \sum_{m \in \mathcal{M}} \sum_{l \in \mathcal{L}} x_{n, m}^{l} \cdot b_{n, m}^{l}-M \cdot C_{\mathrm{SC}}
$$

412 where $C_{\mathrm{SC}}$ is the cost of a SC, corresponding to the sum of its $413 \mathrm{CapEx}$ and the traffic-dependent OpEx. For the calculation of 414 the SCs cost, the cost model presented in [23] is employed.

415 To ensure its objective, the third party introduces a minimum 416 profit, which is described as a function of its total cost, i.e.,

$$
\mathrm{CG}_{\mathrm{SC}}^{\min }=y \cdot M \cdot C_{\mathrm{SC}}, \quad y>0
$$

417 Given the minimum profit, which is calculated by (5), we 418 estimate the reservation price as follows.

419 Proposition 1: The reservation price is defined as the mini420 mum price that a seller would be willing to accept for leasing 421 the corresponding bandwidth, i.e.,

$$
b_{\mathrm{res}, n, m}^{l}=\frac{N_{\mathrm{PRB}_{n, m}}^{l}}{N_{\mathrm{PRB}}^{\max }} \cdot y \cdot M \cdot C_{\mathrm{SC}} .
$$

422 Proof: The third party is willing to share its resources 423 among the MNOs based on proportional fairness by ensuring 424 that a minimum profit gain will be achieved, at the same time. 425 To that end, it calculates a reservation price, which is the 426 minimum price that the third party will accept from an MNO 427 to lease a specific bandwidth. The maximum number of PRBs 428 that the $n$th MNO can offload to the $m$ th $\mathrm{SC}$ is given by

$$
N_{\mathrm{PRB}_{n, m}}^{l}=\frac{b_{\mathrm{res}, n, m}^{l} \cdot x_{n, m}^{l}}{\sum_{n \in \mathcal{N}} \sum_{l \in \mathcal{L}} b_{\mathrm{res}, n, m}^{l} \cdot x_{n, m}^{l}} \cdot N_{\mathrm{PRB}}^{\mathrm{max}}
$$

429 Finally, by using (5) and (7) in (4), the reservation price for 430 offloading traffic is calculated.

431 The reservation price in (6) is not the price that the MNOs 432 will propose to lease their requested capacity, although it is 433 a threshold price that the third party uses to eliminate all the 434 offers that are less than the accepted. The reservation price can 435 be either announced or unknown to the MNOs. The reservation 436 price means that the seller would rather withhold the capacity 437 if the proposed bids are too low (i.e., lower than the reservation 438 price), and given this price, the auction process can be acceler439 ated since the set of prices that are lower than the reservation 440 price can be discarded.

441 2) MNOs' Objective: The objective of each MNO is the 442 maximization of its financial profits. The maximization of the 443 profits of the $n$th MNO is defined as the revenue from switching 444 off its BS minus the winning bids for leasing the requested 445 capacity from the third party. Therefore, the profit (cost gain) 446 can be written as

$$
f_{2}(\mathbf{x})=\mathrm{CG}_{n}=C_{\mathrm{BS}} \cdot x_{n}-\sum_{m \in \mathcal{M}} \sum_{l \in \mathcal{L}} x_{n, m}^{l} \cdot b_{n, m}^{l}
$$

with

$$
x_{n}=\prod_{m \in \mathcal{M}} \sum_{l \in \mathcal{L}} x_{n, m}^{l} \quad \forall n \in \mathcal{N}
$$

and $C_{\mathrm{BS}}$ being the cost of a $\mathrm{BS}$, whose model is also presented 448 in [23], provided that

$$
\sum_{l \in \mathcal{L}} x_{n, m}^{l} \in\{0,1\} \quad \forall n \in \mathcal{N} \quad \forall l \in \mathcal{L}
$$

At this point, let us recall that an operator is able to switch 450 off its BS if it wins in an auction in all the SCs, a condition that 451 is represented by the product in (9). The product is equal to 1452 only when the $n$th MNO wins in $M$ auctions in respective SCs; 453 otherwise, it is 0 .

The MNOs are willing to participate in the auction and lease 455 bandwidth resources from a third party if they guarantee a mini- 456 mum profit gain. Consequently, they introduce a minimum pro- 457 fit, which is described as a percentage of their total costs, i.e., $\quad 458$

$$
\mathrm{CG}_{n}^{\min }=z \cdot C_{\mathrm{BS}}, \quad 0<z<1 .
$$

The bidding strategy of the MNOs, along with the proposed 459 bids, depend on the minimum profit gain and the operation costs 460 of the BSs. However, the cost gain can be attained if and only 461 if the $n$th MNO wins in $M$ auctions and is able to lease the 462 requested capacity. In addition, since we consider uniform traf- 463 fic in the macro cell, we conclude that the bids in the different 464 SCs must be equal and proportional to the corresponding traffic. 465 Thus, the maximum bid price for offloading the traffic in the 466 $m$ th cell is calculated as

$$
b_{n, m}^{L}=\frac{(1-z) \cdot C_{\mathrm{BS}}}{M} .
$$

Similarly, the minimum bidding price is a proportional value 468 of the maximum one, which is given by

$$
b_{n, m}^{0}=v \cdot \frac{(1-z) \cdot C_{\mathrm{BS}}}{M}
$$

where $v \in(0,1)$. The bidding values of the remaining $L-1470$ levels can be calculated based on (3), depending on the maxi- 471 mum bid value and the relation between the outage tolerance of 472 the MNOs and the discount they can be offered for the lower 473 requested bandwidth.

3) Overall Objective: The overall objective of the network 475 is the minimization of the energy consumption. This can be 476 attained by reducing the number of active BSs, given that the 477 BSs are responsible for the major part of energy consumption 478 in the network. The network energy consumption $\mathbb{E}[E]$ is

$f_{3}(\mathbf{x})=\mathbb{E}[E]=\sum_{n \in \mathcal{N}} \mathbb{E}\left[E_{\mathrm{BS}_{n}}\right] \cdot\left(1-x_{n}\right)+M \cdot \mathbb{E}\left[E_{\mathrm{SC}}\right]$

where $\mathbb{E}\left[E_{\mathrm{BS}_{n}}\right]$ and $\mathbb{E}\left[E_{\mathrm{SC}}\right]$ represent the energy consumption 480 of $\mathrm{BS}$ and $\mathrm{SC}$, respectively. 
482 The combinatorial auction formulation should include all the 483 objectives of the participating entities. To capture the trade-off 484 between the objectives, the multiobjective optimization [24] is 485 employed. The target is to find a subset of acceptable solutions 486 according to a set of objectives. In general terms, the objectives 487 may be conflicting, and consequently, a single global optimum 488 may not exist. Hence, the notion of an optimum set $\mathrm{x}^{*}$ becomes 489 very important. Some relevant definitions are given in the 490 following.

491 Definition 1: Given the three objectives, $f_{1}(\mathbf{x}), f_{2}(\mathbf{x})$, and $492 f_{3}(\mathbf{x})$, and provided that $\mathbf{x}_{1}$ and $\mathbf{x}_{2}$ are two decision variables, 493 then $\mathbf{x}_{1}$ is said to be the Pareto dominant, and is denoted $\mathbf{x}_{1} \succeq$ $494 \mathbf{x}_{2}$ if and only if $f_{i}\left(\mathbf{x}_{1}\right) \geq f_{i}\left(\mathbf{x}_{2}\right) \forall i \in\{1,2,3\}$, and $f_{j}\left(\mathbf{x}_{1}\right)>$ $495 f_{j}\left(\mathbf{x}_{2}\right)$, for at least one index $j \in\{1,2,3\}$.

496 Definition 2: $\mathbf{x}^{*}$ is said to be Pareto optimal (or nondom497 inated), if there is no other $\mathbf{x}$, so that $\mathbf{x}$ dominates $\mathbf{x}^{*}$. The 498 set of all Pareto solutions in the decision space is called the 499 Pareto optimal set and the image of the Pareto optimal set in 500 the objective space is called the Pareto optimal front.

AQ3 501 Based on the aforementioned definitions, the ILP multiobjec502 tive optimization problem can be formulated as follows.

503 Definition 3: The allocation problem is to determine the 504 optimal solution $\left\{x_{n, m}^{l}\right\} \forall n \in \mathcal{N} \forall m \in \mathcal{M}$, and $\forall l \in \mathcal{L}$ that 505 maximizes the distinctive objectives of the involved parties in 506 the auction, subject to capacity and offloading targets

P1: $\max \left[\mathrm{CG}_{\mathrm{SC}}, \mathrm{CG}_{n},-\mathbb{E}[E]\right]$

s.t.

$$
\begin{aligned}
& \sum_{n \in \mathcal{N}} \sum_{l \in \mathcal{L}} x_{n, m}^{l} \cdot N_{\mathrm{PRB}_{n, m}}^{l} \leq N_{\mathrm{PRB}}^{\max } \forall m \in \mathcal{M} \\
& \sum_{l \in \mathcal{L}} x_{n, m}^{l} \in\{0,1\} \quad \forall n \in \mathcal{N} \quad \forall m \in \mathcal{M} \\
& b_{n, m}^{l} \geq b_{\mathrm{res}, n, m}^{l} \forall n \in \mathcal{N} \forall m \in \mathcal{M} \forall l \in \mathcal{L} \\
& x_{n, m}^{l} \in\{0,1\} \quad \forall n \in \mathcal{N} \forall \forall \in \mathcal{M} \forall l \in \mathcal{L} .
\end{aligned}
$$

507 The constraint in (16) ensures that the total number of 508 allocated resources does not exceed their availability, constraint 509 (17) ensures that only one bid of the $n$th MNO in the $m$ th SC 510 can be the winning bid among the $l$ different ones, constraint 511 (18) ensures that the bids are higher than the reservation price, 512 and constraint (19) ensures the integrality of the binary variable. 513 The objectives of the proposed optimization formulation are 514 contradictory. The maximization of the third party income does 515 not imply the BSs switching off, whereas the energy objective 516 does not ensure the third party's interests. The maximization 517 of the financial gains of the third party may lead to additional 518 economic losses from the MNOs' perspective since the maxi519 mization of this objective may lead to a resource allocation that 520 does not imply the deactivation of BSs.

521 Exploiting the fact that the BSs are responsible for the major 522 part of the energy consumption, the third objective of the prob523 lem $f_{3}(\mathbf{x})$ can be transformed into a constraint. Energy con524 sumption is minimized when the maximum number of MNOs 525 switches off their BSs after winning in $M$ auctions, a condition 526 represented by the product in (9). Thus, the problem P1 is transformed into a simpler formulation in (20), and at the same 527 time, the energy consumption objective is not neglected, in 528 contrast to former works [17], where only the maximization of 529 the third party's income is considered. The equivalent problem 530 is as follows:

P2: $\max \left[\mathrm{CG}_{\mathrm{SC}}, \mathrm{CG}_{n}\right]$

s.t.

$$
\begin{aligned}
& \prod_{m \in \mathcal{M}} \sum_{l \in \mathcal{L}} x_{n, m}^{l} \in\{0,1\} \quad \forall n \in \mathcal{N} \\
& \sum_{n \in \mathcal{N}} \sum_{l \in \mathcal{L}} x_{n, m}^{l} \cdot N_{\mathrm{PRB}_{n, m}}^{l} \leq N_{\mathrm{PRB}}^{\max } \forall m \in \mathcal{M} \\
& \sum_{l \in \mathcal{L}} x_{n, m}^{l} \in\{0,1\} \quad \forall n \in \mathcal{N} \quad \forall m \in \mathcal{M} \\
& b_{n, m}^{l} \geq b_{\mathrm{res}, n, m}^{l} \forall n \in \mathcal{N} \forall m \in \mathcal{M} \quad \forall l \in \mathcal{L} \\
& x_{n, m}^{l} \in\{0,1\} \quad \forall n \in \mathcal{N} \quad \forall m \in \mathcal{M} \quad \forall l \in \mathcal{L} .
\end{aligned}
$$

The objective function (20) aims at maximizing the financial 532 gain of both the third party and the MNOs. Constraint (21) 533 ensures that an operator either wins in one auction in the $M 534$ SCs (and switches off its BS) or loses in all the auctions (keeps 535 its BS active). Thus, this constraint ensures the minimization 536 of the energy consumption given by (14) since the selected 537 resource allocation leads to the highest number of switched-off 538 BSs, while at the same time achieving the increase in MNOs 539 and third party's income. Constraints (22)-(25) are the same as 540 (16)-(19), respectively.

541

The multiobjective problem P2 belongs to the class 542 NP-Complete since it is equivalent to the 0-1 knapsack prob- 543 lem, which is a well-known problem in combinatorial optimiza- 544 tion. For the NP-hard problems, an optimal solution is difficult 545 to be found due to the large number of variables [24]. However, 546 there are different solutions that represent the best feasible state 547 of the investigated system, e.g., the best resource allocation 548 for the deactivation of the highest number of BSs. To find the 549 best possible representation or a good approximation of these 550 optimal solutions that are widely known as the Pareto optimal 551 set or as the optimal Pareto front and to overcome the high 552 complexity of multiobjective problems, metaheuristic methods 553 (also sometimes called genetics) have become a very active 554 research area, and several algorithms have been proposed [25], 555 [26]. Although, the metaheuristic algorithms do not guarantee 556 that this approximation is the optimal, the solutions are still 557 good and near optimal. ${ }^{4}$

The metaheuristic algorithm that finds the Pareto solution 559 for our multiobjective optimization problem works on a set 560 of possible combinations of the decision variables [24]. The 561 basic definition of the Pareto front is that it consists of exactly 562 those point solutions that are not dominated by any other point. 563 The different objectives cannot be optimized simultaneously. 564 Thus, first, the solutions that maximize the MNOs's income 565 and the third party's economic gains are calculated separately. 566

\footnotetext{
${ }^{4}$ For the sake of simplicity, we use the term "Pareto front" throughout this paper to refer to the obtained solutions through metaheuristics.
} 
567 Obviously, these solutions that maximize the cost gains of the 568 MNOs and the third-party always belong to the Pareto front 569 and, in fact, they are its endpoints. A simple algorithm to 570 find the other solutions (if any) on the Pareto front begins by 571 sorting the solutions according to one of the objectives, e.g., 572 third party's income. The algorithm then starts with the point 573 with the maximum gain for the third party and continues to 574 the successive solutions in order of increasing third party's cost 575 until the solutions with higher cost gain value for the operators 576 is found. This solution is then added to the Pareto front, and the 577 search is restarted from it.

578 Through the use of metaheuristics, the problem can be solved 579 efficiently and quickly for a relatively small number of MNOs 580 and SCs [25], [27], [28]. This assertion cannot be concluded 581 and generalized for large networks. In the investigated network 582 configuration with 5 MNOs, 15 SCs, and 9 bidding levels, the 583 runtime of the solution is very short. At this point, let us clarify 584 that the numbers selected for the MNOs and the SCs are based 585 on the most typical and realistic scenarios in recent studies [18], 586 [19] since the most common scenarios in European countries 587 involve three to four MNOs, and 15 SCs can cover the service 588 are of one macro BS.

\section{D. Pricing Strategy}

590 Having defined the ILP model, we now illustrate the payment 591 rule, which is of crucial importance for the realization of the 592 auction for the third party. The VCG payment induces all 593 the users to reveal their actual valuations for the requested 594 bandwidth. In the VCG mechanism, the third party charges the 595 bidders (MNOs) with a price for the requested capacity. For 596 example, an MNO who requires large bandwidth will have to 597 pay a high price since its request results in dissatisfying other 598 MNOs who lose in the auction because of the limited capacity 599 resources and will not be able to offload their traffic.

600 Let us denote by $p_{n, m}^{l}$ the price paid by the MNO $n$ to the 601 third party for the allocated capacity of the $m$ th SC. The payoff 602 function for bidder $n$ that represents the difference between the 603 gain of the MNO attained through the BS switching off and 604 the true valuation minus the paid price (let us recall that in the 605 truthful auction $u_{n, m}^{l}=b_{n, m}^{l}$ ) is

$$
\mathcal{U}_{i}= \begin{cases}\mathrm{CG}_{i}+\sum_{m \in \mathcal{M}} \sum_{l \in \mathcal{L}} u_{i, m}^{l}-\sum_{m \in \mathcal{M}} \sum_{l \in \mathcal{L}} p_{i, m}^{l}, & \text { if } i \text { is selected } \\ -C_{\mathrm{BS}}, & \text { otherwise. }\end{cases}
$$

606 The payment rule for each MNO can be defined as follows:

$$
\begin{aligned}
p_{i, m}^{l}= & \sum_{n \in \mathcal{N} \backslash\{i\}} \sum_{m \in \mathcal{M}} \sum_{l \in \mathcal{L}}\left(x_{i, m}^{l}\right)^{\{-i\}} \cdot b_{i, m}^{l} \\
- & \left(\sum_{n \in \mathcal{N} \backslash\{i\}} \sum_{m \in \mathcal{M}} \sum_{l \in \mathcal{L}} x_{i, m}^{l} \cdot b_{i, m}^{l}\right. \\
& \left.-\sum_{n \in \mathcal{N} \backslash\{i\}} \sum_{m \in \mathcal{M}} \sum_{l \in \mathcal{L}} x_{i, m}^{l} \cdot b_{i, m}^{l}\right)
\end{aligned}
$$

TABLE I

Simulation PARAMETERS

\begin{tabular}{rl|rl}
\hline Parameter & Value & Parameter & Value \\
\hline \hline Number of MNOs, $N$ & 5 & BS bandwidth, $C R_{B S}$ & $20 \mathrm{MHz}$ \\
Number of SCs, $M$ & 15 & SC bandwidth, $C R_{S C}$ & $5 \mathrm{MHz}$ \\
Traffic load, Load & Fig. 3 & $\delta$ & {$[0.5,1.5]$} \\
\hline
\end{tabular}

where the first term is the aggregate valuation of the allocated 607 resources, $\left(x_{n, m}^{l}\right)^{\{-i\}}$, when MNO $i$ does not participate at all 608 in the auction. The second term is the aggregate valuation of the 609 allocation $x_{n, m}^{l}$ of all MNOs other than $i$, when $i$ participates 610 in the auction. Next, we prove that our optimized auction 611 mechanism possesses two important properties: 1) truthfulness 612 and 2) individual rationality.

Proposition 2 (Truthfulness): The payment rule defined in 614 (27) satisfies the truthfulness property.

615

Proof: The proof is given in Appendix B in the supple- 616 mental file.

Proposition 3 (Individual Rationality): The payment rule 618 defined in (27) satisfies the individual rationality property, and 619 all bidders are guaranteed to obtain nonnegative utility.

Proof: The proof is given in Appendix C in the supple- 621 mental file.

622

\section{Performance Evaluation}

This section is composed of three parts. First, we explain 624 the simulation scenario employed both for the multiobjective 625 optimization and the system-level simulations. Second, we 626 present the results regarding the estimation of the Pareto front 627 for the multiobjective optimization solved by using the Matlab 628 tools. Finally, we show a performance comparison between 629 the proposed scheme and two state-of-the-art schemes with the 630 development of a custom-made $\mathrm{C}$ simulator.

\section{A. Simulation Scenario}

The simulation scenario corresponds to a dense HetNet de- 633 ployment, such as a university campus or an urban area. More 634 specifically, our scenario focuses on a cell served by the BSs of 635 $N=5$ MNOs and $M=15 \mathrm{SCs}$ that cover the whole cell area. 636 In our experiments, we consider various scenarios, wherein the 637 MNOs have different traffic volumes (i.e., $\rho$ ), different bidding 638 strategies (i.e., nontolerant, linear, and tolerant), different cost 639 requirements (i.e., $z$ ), and different bidding levels (i.e., $L$ ). 640 The system-level simulations are based on Monte Carlo exper- 641 iments, and the presented results focus on the night zone for 642 the duration of one year. ${ }^{5}$ The set of parameters is provided 643 in Table I.

To assess the performance of our scheme, we compare 645 the proposed multiobjective auction-base switching-off strat- 646 egy (referred to as MAS), to two benchmark solutions: 1) an 647 auction-based switching-off scheme, wherein the income of the 648

\footnotetext{
${ }^{5}$ Note that the results are calculated for the case of one macro cell, but they can be easily extended to extended configurations. Furthermore, we consider the period of one year since the yearly traffic is considered stable [30].
} 


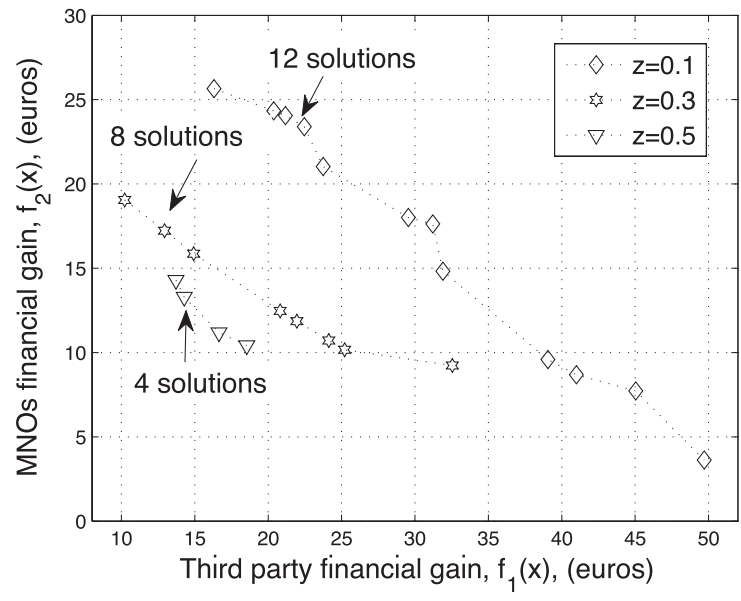

(a)

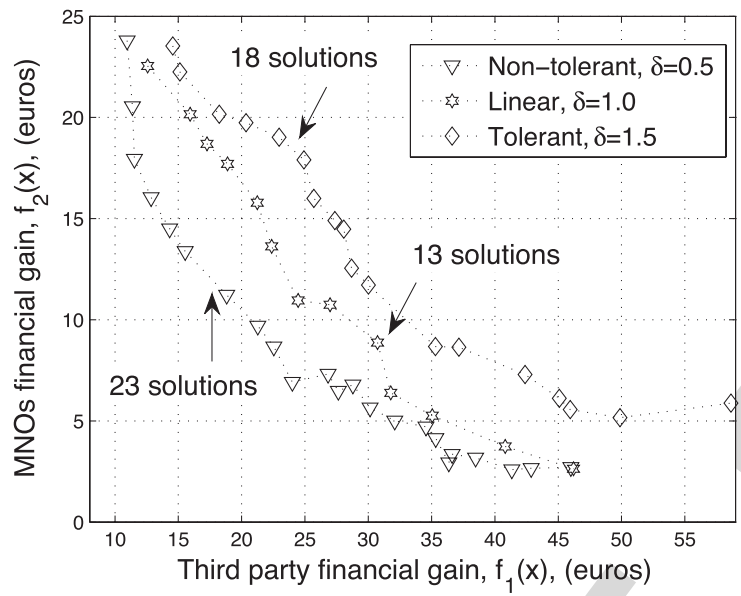

(b)

Fig. 6. Estimation of Pareto front solutions. (a) Estimated Pareto front for multiple MNOs' requirements, $L=2$ and linear bidding strategy. (b) Estimated Pareto front for multiple bidding strategies, $L=2$, and $z=0.1$.

649 third party is the unique objective to be maximized (referred 650 as ISO) [17]; and 2) a baseline scenario with full operational 651 topology (FOT), where none of the BSs is switched off.

\section{B. Estimation of the Pareto Front}

653 Here, we present the Pareto front analysis. In this set of 654 experiments, we assume that all operators have the same traffic 655 volume equal to the maximum possible traffic load, i.e., $\rho_{n}=1$. 656 In addition, we assume that each MNO submits three different 657 bids, corresponding to $L=2$, to the third party.

658 Fig. 6 shows the Pareto front solutions achieved by solving 659 the proposed multiobjective problem for different minimum 660 financial gains with the same bidding strategy [see Fig. 6(a)] 661 and different bidding strategies with the same minimum profit 662 requirement [see Fig. 6(b)]. In both figures, the third party's 663 annual financial gain that can be attained by leasing its re664 sources to one MNO is represented in the $x$-axis, and the annual 665 economic profits of one MNO are given in $y$-axis.

666 In Fig. 6(a), the set of optimal solutions is shown for the 667 linear bidding strategy. Note that the stochastic search per668 formed by means of the metaheuristic algorithm succeeds in estimating a Pareto front for the given parameters of the studied 669 problem. As expected, in all the different cases, the higher the 670 third party's income $\left(f_{1}(\mathbf{x})\right)$, the lower the financial gain of 671 the MNOs $\left(f_{2}(\mathbf{x})\right)$. Thus, the Pareto front is monotonically 672 decreasing, meaning that the improvement of one objective 673 leads to the deterioration of the other objective, and there is 674 no feasible global solution maximizing all the objectives. A 675 second observation relies on the motivation of the MNOs to 676 maximize their economic gains, which is a fact that can be 677 explored through the parameter $z$ that reflects the requirements 678 of the minimum profit gain, denoted in (11). As it is plotted 679 in the figure, the greedy behavior of the MNOs for higher cost 680 gains $(z=0.5)$ leads to lower income for the third party and, 681 at the same time, a lower number of achievable solutions. This 682 result is of significant importance since a greedy behavior from 683 the MNOs' perspective will produce lower bids that may not be 684 accepted by the third party; thus, the MNOs will not be able to 685 offload their whole traffic. The selection of a proper value of the 686 parameter $z$ helps the operators to decide whether it is profitable 687 for them to bid by taking into account their financial needs, 688 and by observing Fig. 6(a), we conclude that lower values of 689 $z$ lead to higher income for the two involved parties. To provide 690 further insights for our approach, let us also highlight that, in 691 a realistic scenario with a large number of macro BSs, the cost 692 gains for the MNOs and the third party are significantly higher. 693 For example, by applying the proposed switching off technique 694 to the central area of London where an MNO may have up to 695 500 deployed BSs [31], the economic gains may reach up to 696 $\sim 12.500 €$ for the MNO and up to $\sim 25.000 €$ for the third 697 party.

In continuation, Fig. 6(b) illustrates the Pareto front by 699 examining different bidding strategies for the case of $z=0.1 .700$ Again, we observe that the attained solutions are decreasing 701 monotonically. Moreover, as we see, the more tolerant the 702 bidding strategy of the MNOs, the higher financial gains for the 703 involved parties. This important insight can be easily explained 704 by taking into account the fact that a nontolerant MNO is more 705 greedy; thus, it requests more capacity for the same bids with 706 respect to a tolerant MNO, as shown in Fig. 5. As a result, 707 the third party may lose its incentive to lease the bandwidth, 708 and the economic benefits will be reduced for the MNOs and 709 the SC network. It is noted that the tolerant bidding strategy 710 offers better solutions due to the leasing of more resources and 711 the switching off of higher number of BSs, whereas the linear 712 bidding strategy implies an intermediate solution between the 713 extreme cases. Hence, along with the minimum guaranteed 714 profit, the bidding strategy is also an important indicator that 715 affects the decision of MNOs on bidding.

\section{Numerical Results}

Here, the performance results with regard to 718 telecommunication-oriented (network throughput and energy 719 efficiency) and cost-oriented (annual cost and income gains) 720 metrics are shown. In this set of experiments, we study a more 721 realistic scenario, where the MNOs have different traffic vol- 722 umes and, thus, different requirements and outcomes, when 723 our proposed algorithm is applied. According to recent studies 724 


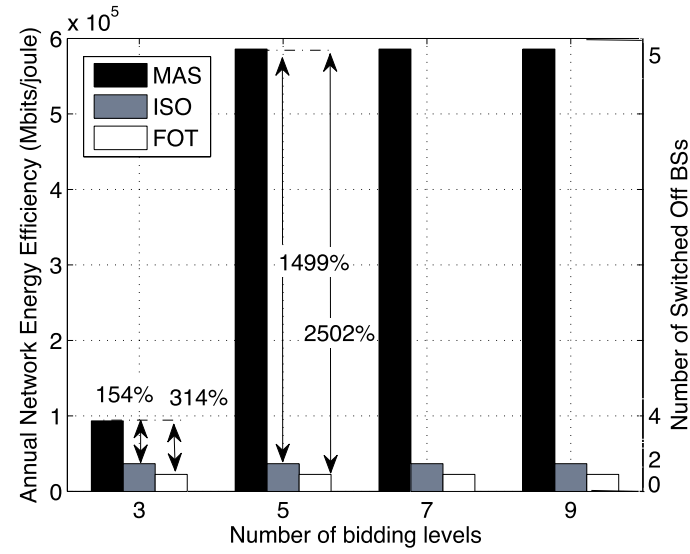

(a)

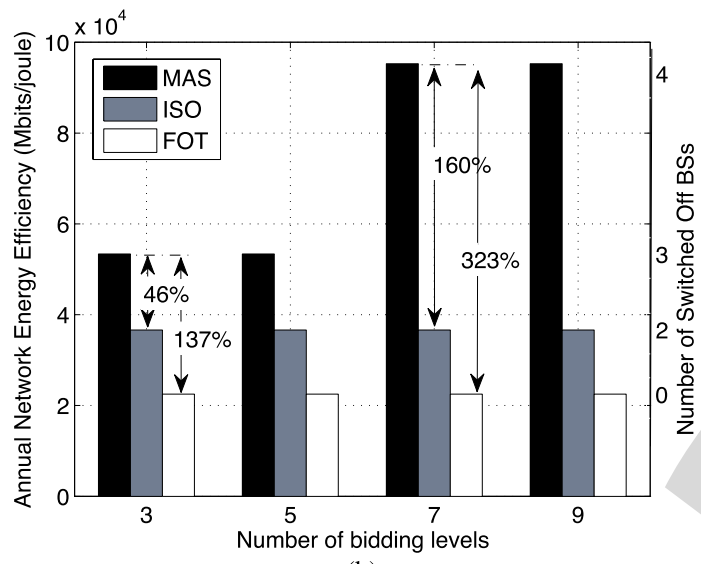

(b)

Fig. 7. Annual energy efficiency for $z=0.1$ and different bidding strategies (a) Tolerant bidding strategy. (b) Nontolerant bidding strategy.

725 [18], in most European countries, there are usually up to 726 two telecommunication companies holding the major part 727 of the market share, up to two smaller operators serving an 728 intermediate portion of the users, and finally, up to one smaller 729 MNO with a very small slice of the market. By exploiting these 730 interesting findings, we consider the scenario in which two 731 MNOs (i.e., $\mathrm{BS}_{1}$ and $\left.\mathrm{BS}_{2}\right)$ are fully loaded $\left(\rho_{1}=\rho_{2}=1.0\right)$, 732 two operators (i.e., $\mathrm{BS}_{3}$ and $\mathrm{BS}_{4}$ ) have relatively lower traffic 733 with $\rho_{3}=\rho_{4}=0.7$, and the fifth $\mathrm{MNO}$ (denoted by $\mathrm{BS}_{5}$ ) 734 has very low traffic $\left(\rho_{5}=0.3\right)$. The results presented in the 735 following are calculated for an operating point on the Pareto 736 front that is assumed to satisfy all the involved parties in the 737 auction. We would like to highlight that the selection of a 738 solution among the Pareto front solutions can be either derived 739 through agreements between the involved parties or another 740 distinguished entity may select it.

741 1) Telecommunication Metrics: Fig. 7 presents the total 742 network energy efficiency versus $L$ for tolerant [see Fig. 7(a)] 743 and nontolerant [see Fig. 7(b)] bidding strategies. The number 744 of switched-off BSs of every algorithm is also shown in the 745 plot (right $y$-axis), along with the percentage gains in terms 746 of energy efficiency of our approach compared with the state747 of-the-art works. First, we observe that the energy efficiency 748 achieved by the MAS scheme increases with the number of 749 levels for tolerant and nontolerant bidding since the biggest

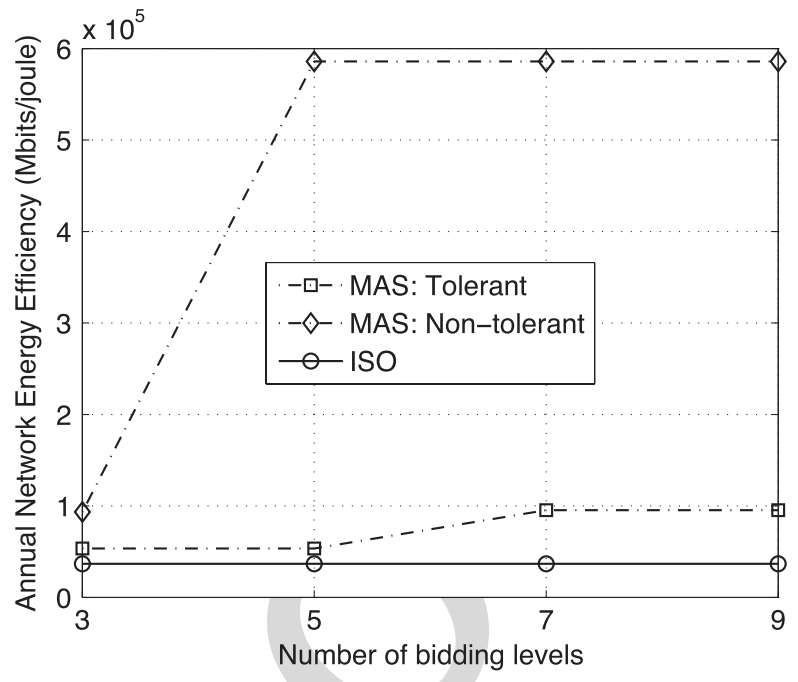

Fig. 8. Annual energy efficiency for different bidding levels and strategies.

variety of the proposed bids (higher number of levels) implies 750 a higher number of choices for the resource allocation. Another 751 important remark is that MAS significantly outperforms the 752 baseline scenario, FOT, (where no BS is switched off), and 753 the ISO algorithm. The better performance of our proposal in 754 terms of energy efficiency is justified by the higher number 755 of switched-off BSs as this is highlighted also in the plots. 756 As we have already mentioned in Section III-C, the goal of 757 our proposal lies in maximizing the energy efficiency, an ob- 758 jective neglected in the auction-based works of the literature, 759 whereby only the maximization of the third party's income 760 was considered. Comparing the behavior of our algorithm in 761 the two figures, it can be observed that the tolerant bidding 762 strategy achieves more considerable gains with respect to the 763 nontolerant bidding, mainly due to the deactivation of many 764 underutilized BSs in the network. These results emphasize 765 the role of the bidding strategy and the number of bidding 766 levels on the achievable energy efficiency, thus providing the 767 necessary insights to the MNOs to select the most appropriate 768 strategy based on their interests. In Fig. 8, the conclusions of 769 Fig. 7(b) and (a) are better illustrated since the comparison 770 between the three schemes and the different bidding strategies 771 is more clearly shown. Finally, we should mention that the 772 results of linear bidding are not shown here for simplicity, given 773 that the achieved gains range between the two aforementioned 774 cases.

Along with the total network energy efficiency performance, 776 it is interesting to study the individual energy efficiency gains 777 of the different MNOs. To that end, the individual gains for 778 the specific (but representative) case of $z=0.1$ and the two 779 different bidding strategies are quantified in Table II, where 780 interesting conclusions can be extracted. In particular, inde- 781 pendently of the bidding strategy and the number of $L$, the 782 ISO scheme is beneficial only for the group of operators that 783 switch off their BSs, who are always the same $\left(\mathrm{MNO}_{1}\right.$ and 784 $\mathrm{MNO}_{2}$ ), whereas the rest of the operators always keep their 785 BSs active. More specifically, the MNOs that switch off their 786 BSs theoretically achieve infinite energy efficiency, as they 787 
TABLE II

Operator ENERgy EFFiciency (X $10^{4}$ Mbits/Joule) With ResPect to the FOT SCHEME FOR $z=0.1$

\begin{tabular}{|c||ccccc|ccccc|}
\hline \multicolumn{1}{|c|}{} & \multicolumn{5}{c|}{ Tolerant bidding } & \multicolumn{5}{c|}{ Non-tolerant bidding } \\
\hline \hline Algorithm & MNO $_{\mathbf{1}}$ & $\mathbf{M N O}_{\mathbf{2}}$ & $\mathbf{M N O}_{\mathbf{3}}$ & $\mathbf{M N O}_{\mathbf{4}}$ & $\mathbf{M N O}_{\mathbf{5}}$ & MNO $_{\mathbf{1}}$ & $\mathbf{M N O}_{\mathbf{2}}$ & $\mathbf{M N O}_{\mathbf{3}}$ & $\mathbf{M N O}_{\mathbf{4}}$ & $\mathbf{M N O}_{\mathbf{5}}$ \\
\hline \hline MAS-L $=\mathbf{3}$ & $\infty$ & 3 & $\infty$ & $\infty$ & $\infty$ & $\infty$ & 3 & 2.2 & $\infty$ & $\infty$ \\
\hline ISO-L $=\mathbf{3}$ & $\infty$ & $\infty$ & 2.2 & 2.2 & 1 & $\infty$ & $\infty$ & 2.2 & 2.2 & 1 \\
\hline \hline MAS-L $=\mathbf{7}$ & $\infty$ & $\infty$ & $\infty$ & $\infty$ & $\infty$ & $\infty$ & 3 & $\infty$ & $\infty$ & $\infty$ \\
\hline ISO-L $=\mathbf{7}$ & $\infty$ & $\infty$ & 2.2 & 2.2 & 1 & $\infty$ & $\infty$ & 2.2 & 2.2 & 1 \\
\hline
\end{tabular}

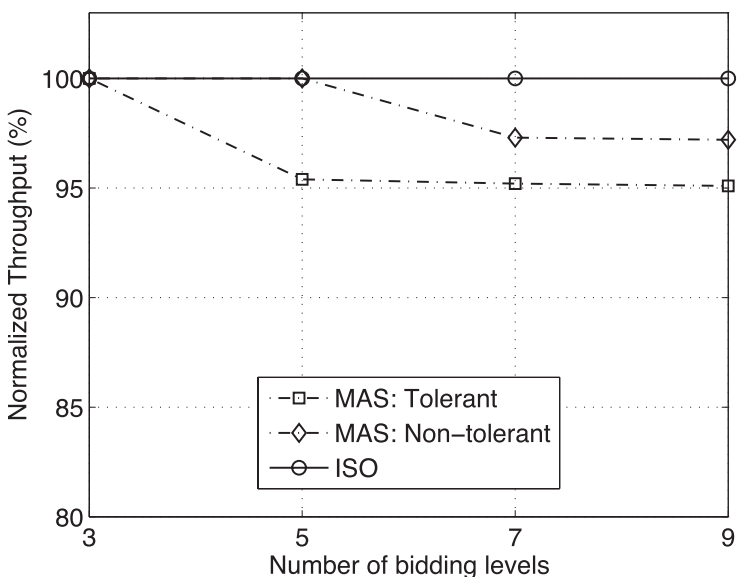

(a)

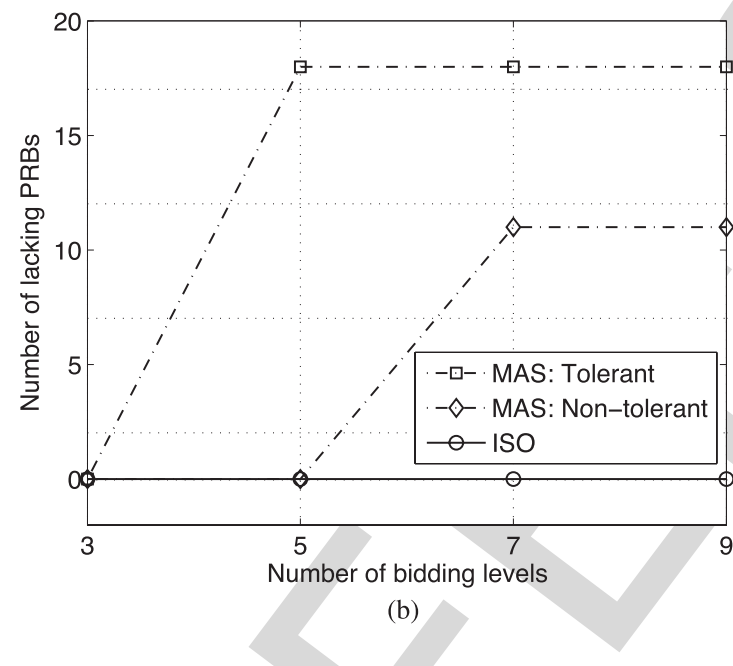

Fig. 9. (a) Normalized throughput for different bidding levels and strategies. (b) Number of lacking PRBs.

788 have their traffic served at zero energy cost, whereas the active 789 operators serve their own traffic without improving their situa790 tion. The proposed MAS eliminates this unfairness by offering 791 the chance to more MNOs to switch off their BSs, providing 792 them with extra incentives to participate in the auction. The 793 individual gains are remarkable of our proposal for both the 794 MNOs and the whole network.

795 Despite the importance of the energy efficiency results, the 796 deactivation of the BSs in the network and the traffic offloading 797 to the SCs potentially implies loss of connections. To that 798 end, we study the normalized throughput that represents the 799 percentage of served connections in the system. In Fig. 9(a), 800 it can be observed that, as the number of switched-off BSs 801 increases, the MAS approach experiences small losses (around
5\% and 3\% for tolerant and nontolerant bidding, respectively). 802 The degraded performance is explained by the high number 803 of deactivated BSs, leading to the service of all the traffic 804 mainly by the SC network (since at most one BS remains 805 active). The MNOs are able to decide whether the throughput 806 performance can be sacrificed to achieve energy efficiency, or 807 even small losses are prohibitive (thus, a tolerant strategy is not 808 acceptable). The results in terms of lacking PRBs are given 809 in Fig. 9(b). As it is observed, a larger number of PRBs are 810 lacking, when the MAS strategy is applied. In our case, there 811 are different reasons for the degraded throughput performance. 812 The unserved users exist because either lower bids (thus, fewer 813 PRBs) are selected or the overall PRBs are not adequate for the 814 total offloaded traffic. However, the impact on the throughput is 815 negligible, as it was shown in Fig. 9(a).

816

2) Cost Metrics: The total annual network cost and the 817 individual annual gains for the operators and the third party, 818 compared with the state-of-the-art algorithm and having as a 819 benchmark the FOT scheme, are presented in Figs. 10 and 12, 820 respectively.

The annual network cost is given versus the different number 822 of bidding levels and for the tolerant [see Fig. 10(a)] and 823 nontolerant [see Fig. 10(b)] bidding strategies, respectively. 824 The total network cost is the sum of the average cost of all 825 MNOs and the third party for the operation of all infrastructures 826 (i.e., the active BSs and SCs). The first observation is that the 827 network cost is the same for level pairs $(l=3$ and $l=5)$ and 828 $(l=7$ and $l=9)$ for the MAS algorithm, due to the number 829 of switched-off BSs that is the same for the two cases. The 830 MAS scheme achieves a considerable reduction up to $94 \% 831$ and $96 \%$ for tolerant bidding, when compared with ISO and 832 FOT schemes, respectively. For the case of nontolerant bidding 833 strategy, the cost reduction is lower. This result is explained by 834 the fact that MNOs are more greedy, and in their attempt to 835 increase their economic gains, they do not offer high bids for 836 the requested capacity. As a consequence, the third party does 837 not accept the allocation of resources to low prices, and some 838 BSs may not be switched off. Concerning the ISO scheme, we 839 observe that the total annual cost is not affected by the different 840 bidding levels since, in ISO auction, only one bid is offered 841 based on the maximum traffic expectations, and fewer BSs are 842 deactivated. A clear comparison between MAS, for tolerant and 843 nontolerant bidding, and ISO is given in Fig. 11, where the 844 annual network cost behavior is highlighted.

The individual revenue of each MNO and the third party is 846 plotted in Fig. 12. We may observe that, similar to the energy 847 efficiency gains, the ISO scheme provides financial gains only 848 to particular operators $\left(\mathrm{MNO}_{1}\right.$ and $\left.\mathrm{MNO}_{2}\right)$ and particularly to 849 


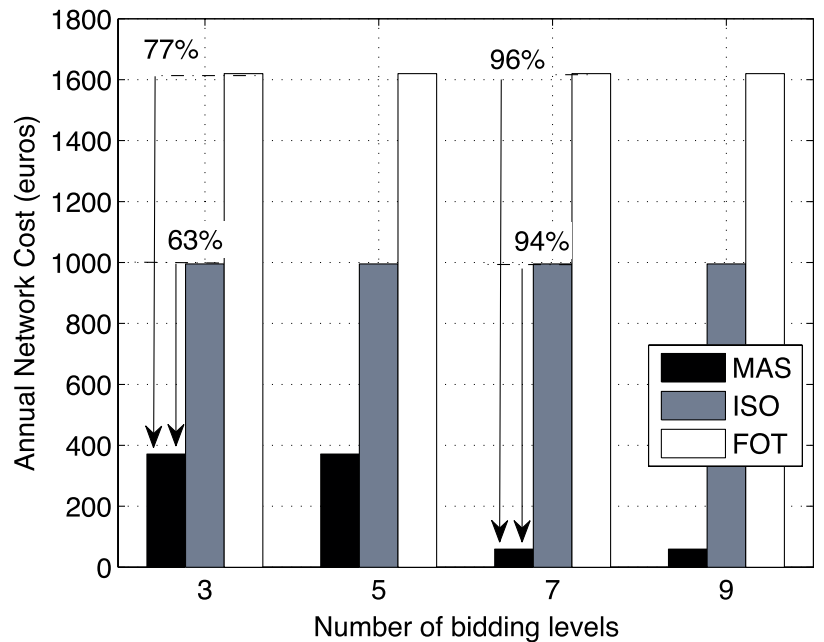

(a)

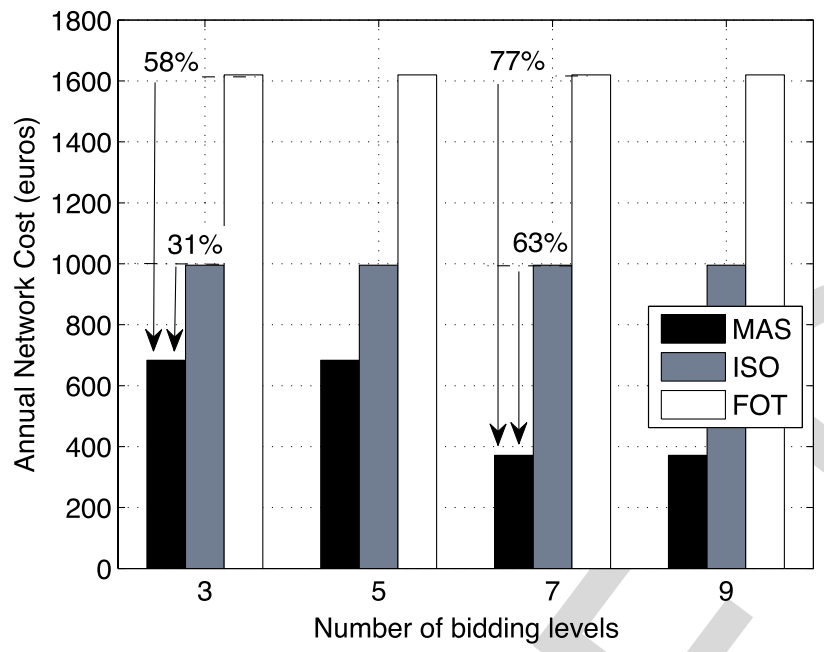

(b)

Fig. 10. Annual network cost for $z=0.1$ and different bidding strategies (a) Tolerant bidding strategy. (b) Nontolerant bidding strategy.

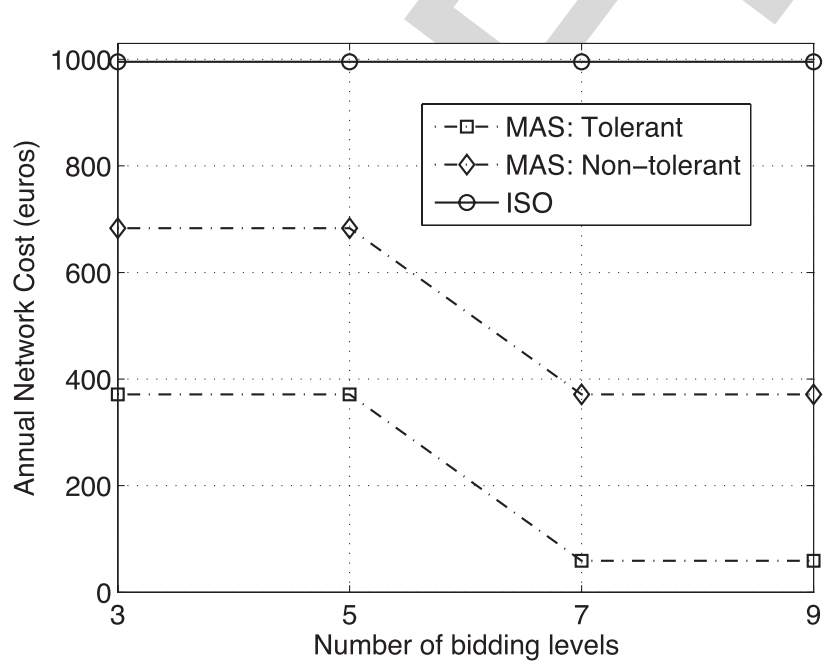

Fig. 11. Annual network cost for different bidding levels and strategies.

850 the MNOs that switch off their networks, whereas the economic 851 gains of the remaining operators are zero compared with the 852 FOT scheme since these MNOs keep their BSs active and serve

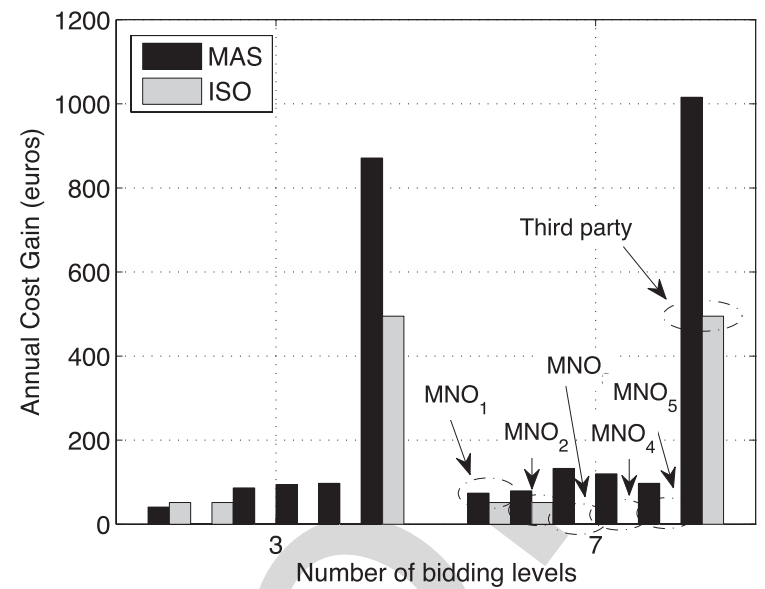

(a)

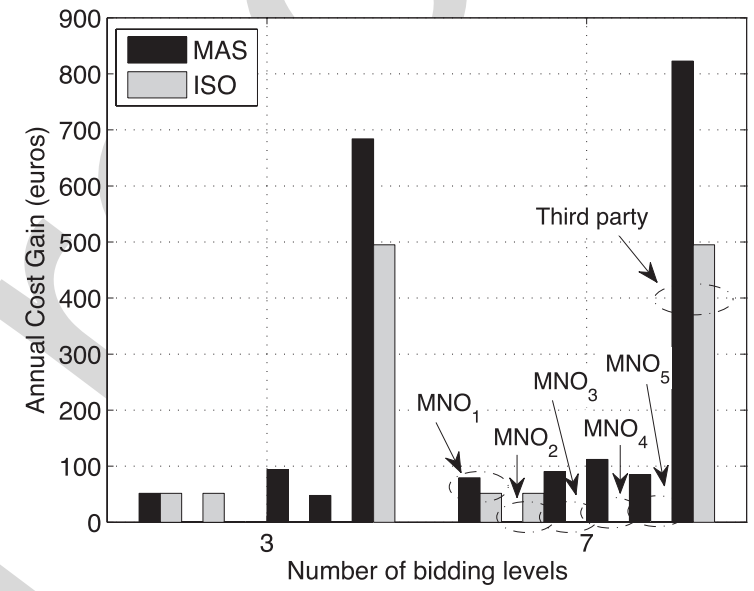

(b)

Fig. 12. Annual cost gain for $z=0.1$ and different bidding strategies. (a) Tolerant bidding strategy. (b) Nontolerant bidding strategy.

their own traffic without having any benefit from the auction. 853 On the other hand, for the proposed MAS approach, more 854 operators are able to have economic benefits, independently of 855 the particular number of levels. Furthermore, as the number of 856 bidding levels increases, the MAS algorithm achieves higher 857 financial gains for the operators due to the higher number 858 of combinations of bids offered. In addition, for the tolerant 859 bidding strategy [see Fig. 12(a)], the economic gains are higher 860 compared with the nontolerant behavior [see Fig. 12(b)].

\section{Discussion}

Based on the analysis in Sections IV-B and IV-C, we have 863 shown that the proposed MAS scheme outperforms the state-of- 864 the-art approaches in terms of energy efficiency (network and 865 individual), annual network cost, and individual cost gains. In 866 addition, it achieves balanced results for the MNOs with respect 867 to the individual energy efficiency and cost gains. Furthermore, 868 through a performance assessment, we have identified the sig- 869 nificance of the bidding behavior, the number of levels, and pa- 870 rameter $z$. In particular, better performance results are attained 871 in terms of energy efficiency, aggregate network cost, and indi- 872 vidual cost gains, when a higher number of levels are chosen 873 
874 and through tolerant bidding. Moreover, higher values of $z$ 875 achieve lower gains for both the MNOs and the third party. Fi876 nally, although minor losses may appear in terms of throughput, 877 the attained benefits with respect to energy efficiency, individ878 ual and network cost gains are remarkable and are adequate for 879 giving the necessary incentives to the MNOs and the third party 880 to apply the proposed auction-based switching-off strategy.

\section{1}

\section{CONCLUSION}

882 In this paper, motivated by the low BS utilization during 883 the night and the coexistence of multiple MNOs and third884 party SCs in the same area, we proposed a novel auction-based 885 offloading and switching-off algorithm that achieves energy 886 savings and cost reduction by encouraging MNOs to offload 887 their traffic and switch off the redundant BSs. Moreover, by 888 employing auction tools and novel bidding strategies, we intro889 duced a switching-off scheme that allows the MNOs to reduce 890 their expenditures. The proposed scheme has been evaluated 891 in terms of energy efficiency and cost metrics for various 892 conditions (different bidding levels and behaviors). The results 893 have shown that our proposal can significantly improve the 894 network energy efficiency, guaranteeing at the same time the 895 throughput. Regarding the financial costs/gains, the proposed 896 scheme provides higher cost benefits and fairness compared 897 with the state-of-the-art algorithms. It provides also interesting 898 insights concerning the rules and behaviors that the MNOs 899 should follow when they participate in an auction strategy. The 900 study of other schemes for the solution of the problem (such 901 as backwards induction) and the potential trade-offs would be 902 interesting research line for future works.

\section{REFERENCES}

1] "Cisco visual networking index: Global mobile data traffic forecast update," Cisco, San Francisco, CA, USA, pp. 2014-2019, Feb. 2015.

[2] X. Bao, Y. Lin, U. Lee, I. Rimac, and R. R. Choudhury, "Dataspotting: Exploiting naturally clustered mobile devices to offload cellular traffic," in Proc. IEEE INFOCOM, Apr. 2013, pp. 420-424

[3] "Nokia networks: Solutions," Nokia, Espoo, Finland, 2015. [Online] Available: http://networks.nokia.com/portfolio/solutions/heterogeneousnetworks

[4] L. Gao, G. Iosifidis, H. Jianwei, and L. Tassiulas, "Economics of mobile data offloading," in Proc. IEEE Workshop INFOCOM, Apr. 2013, pp. 3303-3308.

[5] W. Dong et al. "iDEAL: Incentivized dynamic cellular offloading via auctions," in Proc. IEEE INFOCOM, Apr. 2013, pp. 755-763.

[6] S. Paris, F. Martignon, I. Filippini, and A. Capone, "A truthful auction for access point selection in heterogeneous mobile networks," in Proc. IEEE ICC, Jun. 2012, pp. 3200-3205

[7] X. Zhuo, W. Gao, G. Cao, and S. Hua, "An incentive framework for cellular traffic offloading," IEEE Trans. Mobile Comput., vol. 13, no. 3, pp. 541-555, Mar. 2014.

8] G. Fettweis and E. Zimmermann, "ICT energy consumption-trends and challenges," in Proc. WPMC, Sep. 2008, pp. 1-4.

[9] G. Auer et al., "How much energy is needed to run a wireless network?" IEEE Wireless Commun., vol. 18, no. 5, pp. 40-49, Oct. 2011.

0] F. Han, Z. Safar, and K. J. R. Liu, "Energy-efficient base-station cooperative operation with guaranteed QoS," IEEE Trans. Commun., vol. 61, no. 8, pp. 3505-3517, Aug. 2013.

1] E. Oh, K. Son, and B. Krishnamachari, "Dynamic base station switchingon/off strategies for green cellular networks," IEEE Trans. Wireless Commun., vol. 12, no. 5, pp. 2126-2136, May 2013.

12] A. Bousia, E. Kartsakli, L. Alonso, and C. Verikoukis, "Dynamic energy efficient distance-aware base station switch on/off scheme for LTEAdvanced," in Proc. IEEE GLOBECOM, Dec. 2012, pp. 1532-1537.
[13] A. Bousia, E. Kartsakli, A. Antonopoulos, L. Alonso, and C. Verik- 936 oukis, "Game theoretic infrastructure sharing in multi-operator cellular 937 networks," IEEE Trans. Veh. Technol., DOI: 10.1109/TVT.2015.2445837, 938 to be published.

[14] J. Bartelt, A. Fehske, H. Klessig, G. Fettweis, and J. Voigt, "Joint band- 940 width allocation and small cell switching in heterogeneous networks," 941 in Proc. IEEE VTC-Fall, Sep. 2013, pp. 1-5.

[15] Y. S. Soh, T. Q. S. Quek, M. Kountouris, and H. Shin, "Energy efficient 943 heterogeneous cellular networks," IEEE J. Sel. Areas Commun., vol. 31, 944 no. 5, pp. 840-850, May 2013.

[16] M. H. Alsharif, R. Nordin, and M. Ismail, "A review on intelligent base 946 stations cooperation management techniques for greener LTE cellular 947 networks," J. Commun., vol. 9, no. 12, pp. 937-945, Dec. 2014.

[17] S. Paris, F. Martignon, I. Filippini, and L. Chen, "A bandwidth trad- 949 ing marketplace for mobile data offloading," in Proc. IEEE INFOCOM, 950 Apr. 2013, pp. 430-434.

[18] M. A. Marsan and M. Meo, "Network sharing and its energy bene- 952 fits: A study of European mobile network operators," in Proc. IEEE 953 GLOBECOM, Dec. 2013, pp. 2561-2567.

[19] "Small cell market status," Small Cell Forum, U.K., Feb. 2013. [Online]. 955 Available: http://www.smallcellforum.org/resources-reports 956

[20] "3rd generation partnership project; technical specification group services 957 and system aspects; telecommunication management; study on Energy 958 Savings Management (ESM) (Release 10)," Third-Generation Partnership 959 Project, Sophia Antipolis Cedex, France, Tech. Rep., 3GPP TR 32.826, 960 V10.0.0, Mar. 2010.

[21] "Mobile traffic forecasts 2010-2020 Report," UMTS Forum, London, 962 U.K., Jan. 2011.

[22] V. Krishna, Auction Theory. San Diego, CA, USA: Academic, 2010. 964

[23] A. Bousia, E. Kartsakli, A. Antonopoulos, L. Alonso, and C. Verikoukis, 965 "Sharing the small cells for energy efficient networking: How much does 966 it cost?" in Proc. IEEE GLOBECOM, Dec. 2014, pp. 2649-2654. 967

[24] Y. Sawaragi, I. Hirotaka, and T. Tanino, Theory of Multiobjective Opti- 968 mization, 1st ed. New York, NY, USA: Academic, 1985.

[25] T. Weise, Global Optimization Algorithms-Theory and Application, 2nd 970 ed. Hefei, China: Thomas Weise, 2009. [Online]. Available: http://www. 971 it-weise.de/

[26] R. L. Carraway, T. L. Morin, and H. Moskowitz, "Generalized dynamic 973 programming for multicriteria optimization," Eur. J. Oper. Res., vol. 44, 974 no. 1, pp. 95-104, Jan. 1990.

[27] H. G. Beyer and K. Deb, "On self-adaptive features in real-parameter 976 evolutionary algorithms," IEEE Trans. Evol. Comput., vol. 5, no. 3, 977 pp. 250-270, Jun. 2001.

[28] C. A. Coello, G. B. Lamont, and D. A. Van Veldhuizen, Evolutionary Al- 979 gorithms for Solving Multi-Objective Problems, ser. Genetic and Evolu- 980 tionary Computation, 2nd ed. Berlin, Germany: Springer-Verlag, 2007. 981

[29] L. M. Schmitt, "Theory of genetic algorithms II: Models for genetic oper- 982 ators over the string-tensor representation of populations and convergence 983 to global optima for arbitrary fitness function under scaling," Theoretical 984 Comput. Sci., vol. 310, pp. 181-231, 2004.

[30] "Ericsson mobility report: On the pulse of the networked society," 986 Ericsson, Stockholm, Sweden, Tech. Rep. Nov. 2014.

[31] "Sitefinder: Mobile Phone Base Station Database," Ofcom, London, 988 U.K., Mar. 2015. [Online]. Available: http://sitefinder.ofcom.org.uk/search 989

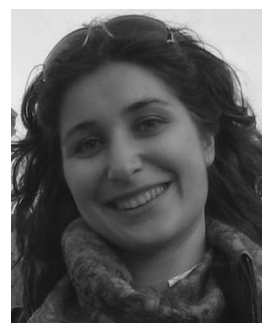

Alexandra Bousia (M'15) received the B.S. and 990 M.S. degrees from the University of Thessaly, Volos, 991 Greece, in 2008 and 2009, respectively. She is cur- 992 rently working toward the $\mathrm{Ph} . \mathrm{D}$. degree in wireless 993 communications with the Department of Signal The- 994 ory and Communications, Universitat Politècnica de 995 Catalunya (UPC), Barcelona, Spain. 996

She is also currently a Marie Curie Researcher 997 with UPC. Since 2011, she has been involved in 998 several national and European projects. Her research 999 interests include wireless networks, medium-access- 1000 control protocols, energy-efficient protocols, and radio resource management 1001 algorithms.

Ms. Bousia received the Best Paper Award at the IEEE Global Communica- 1003 tions Conference in 2014. 


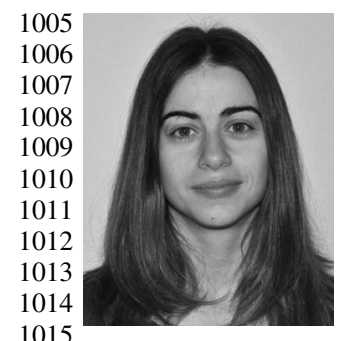

Elli Kartsakli (SM'15) received the Bachelor's degree in electrical and computer engineering from the National Technical University of Athens, Athens, Greece, in 2003; the M.Sc. degree in mobile and satellite communications from the University of Surrey, Surrey, U.K., in 2004; and the Ph.D. degree in wireless telecommunications from the Universitat Politècnica de Catalunya (UPC), Barcelona, Spain, in 2012.

She is currently a Postdoctoral Researcher with UPC. She has participated in several national and 1016 European projects (e.g., GREENET and WSN4QoL). Her main research inter1017 ests include wireless networking, channel access protocols, and energy-efficient 1018 protocols.

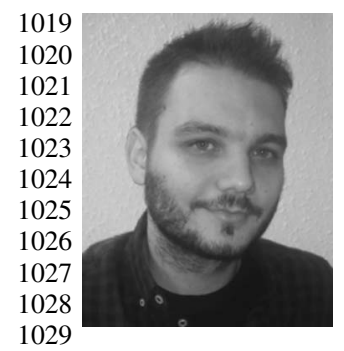

Angelos Antonopoulos (SM'15) received the Ph.D. degree from the Universitat Politècnica de Catalunya, Barcelona, Spain, in 2012.

$\mathrm{He}$ is currently with the Telecommunications Technological Center of Catalonia, Barcelona, Spain. $\mathrm{He}$ has participated in several European projects. $\mathrm{He}$ is the author of more than 50 papers on various topics, including cooperative communications, network coding, and green networking.

Dr. Antonopoulos has received an award from the Technical Chamber of Greece for Exceptional Grad1030 uate Performance. In 2014, he also received the Best Paper Award at the IEEE 1031 Global Communications Conference and the Best Demo Award at the IEEE 1032 International Workshop on Computer-Aided Modeling Analysis and Design of 1033 Communication Links and Networks. In January 2015, he was nominated as 1034 Exemplary Reviewer for the IEEE COMMUNICATIONS LETTERS.

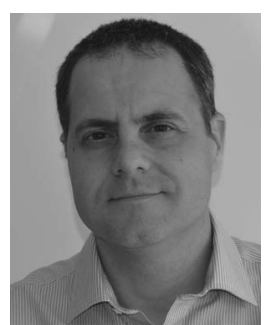

Luis Alonso (SM'XX) received the Ph.D. de- 1035 AQ6 gree from the Universitat Politècnica de Catalunya, 1036 Barcelona, Spain, in 2001.

Since 2006, he has been an Associate Professor 1038 with UPC. He is a Cofounder of the Wireless Com- 1039 munications and Technologies Research Group 1040 (WiComTec). He is the author of 40 research papers, 1041 one book, 12 book chapters, and more than 100 papers 1042 in international congresses. His research interests 1043 include medium access protocols, cross-layer opti- 1044 mization, cooperative transmissions, cognitive radio, 1045 and quality of service for all kinds of wireless networks.

1046

Dr. Alonso has received several best paper awards.

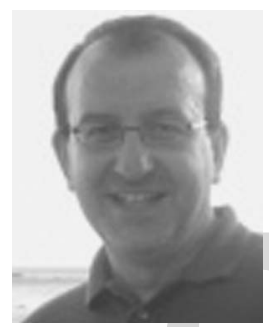

Christos Verikoukis (SM'07) received the Ph.D. de- 1048 gree from the Universitat Politècnica de Catalunya, 1049 Barcelona, Spain, in 2000.

1050

He is currently a Senior Researcher with the Tele- 1051 communications Technological Center of Catalo- 1052 nia, Barcelona, and an Adjunct Professor with 1053 the University of Barcelona. He is the author or 1054 coauthor of 86 journal papers, over 160 conference 1055 papers, three books, 14 book chapters, and two 1056 patents. He has participated in more than $30 \mathrm{com}-1057$ petitive projects and has served as a principal in- 1058 vestigator of national projects. He has supervised $15 \mathrm{Ph} . \mathrm{D}$. students and five 1059 postdoctoral researchers.

1060

Dr. Verikoukis currently serves as the Chair of the IEEE Communication 1061 Society Communications Systems Integration and Modeling Technical Com- 1062 mittee. He received the Best Paper Award at the IEEE International Conference 1063 on Communications in 2011 and the IEEE Global Communications Conference 1064 in 2014 and 2015, as well as the EURASIP 2013 Best Paper Award for the 1065 Journal on Advances in Signal Processing. 


\section{AUTHOR QUERIES}

\section{AUTHOR PLEASE ANSWER ALL QUERIES}

$\mathrm{AQ} 1$ = Please check if the expanded form provide for "PRBs" is appropriate. If not, kindly provide the necessary correction.

AQ2 = If possible, please provide an updated image of Fig. 5 so that the labels for the $\mathrm{x}$ - and $\mathrm{y}$-axis are enlarged for improved readability.

AQ3 = Please expand the ILP acronym.

AQ4 = Please update the images of Figs. 6(a) and (b) so that the x-axis label is amended to reflect "Thirdparty..." instead of "Third party..."

AQ5 = Please provide publication update in Ref. [13].

AQ6 = Please provide membership history of author "Luis Alonso."

\section{END OF ALL QUERIES}




\title{
Multiobjective Auction-Based Switching-Off Scheme 2 in Heterogeneous Networks: To Bid or Not to Bid?
}

\author{
Alexandra Bousia, Member, IEEE, Elli Kartsakli, Senior Member, IEEE, \\ Angelos Antonopoulos, Senior Member, IEEE, Luis Alonso, Senior Member, IEEE, and \\ Christos Verikoukis, Senior Member, IEEE
}

6 Abstract-The emerging data traffic demand has caused a mas7 sive deployment of network infrastructure, including macro base 8 stations (BSs) and small cells (SCs), leading to increased energy 9 consumption and expenditures. However, the network underuti10 lization during low traffic periods (e.g., night zone) enables the 11 mobile network operators (MNOs) to save energy by having their 12 traffic served by third-party SCs, thus being able to switch off 13 their BSs. In this paper, we propose a novel market approach to 14 foster the opportunistic utilization of the unexploited SCs capacity, 15 where the MNOs, instead of requesting the maximum capacity 16 to meet their highest traffic expectations, offer a set of bids re17 questing different amounts of resources from the third-party SCs 18 at lower costs. Motivated by the conflicting financial interests of 19 the MNOs and the third party, the restricted capacity of the SCs 20 that is not adequate to carry the whole traffic in multioperator 21 scenarios, and the necessity for energy-efficient solutions, we in22 troduce a combinatorial auction framework, which includes 1) a 23 bidding strategy, 2) a resource-allocation scheme, and 3) a pricing 24 rule. We propose a multiobjective framework as an energy- and 25 cost-efficient solution for the resource-allocation problem, and we 26 provide extensive analytical and experimental results to estimate 27 the potential energy and cost savings that can be achieved. In 28 addition, we investigate the conditions under which the MNOs 29 and the third-party companies should take part in the proposed 30 auction.

31 Index Terms-Auction, energy efficiency, game theory, green 32 networking, heterogeneous networks (HetNets), multiobjective op33 timization, offloading, switching off.

34

\section{A. Motivation}

36 The rapid expansion of mobile services, along with the 37 emerging demand for multimedia applications, driven by the 38 widespread use of laptops, tablets, and smart devices, has led

Manuscript received April 22, 2015; revised August 7, 2015, October 5, 2015, and December 24, 2015; accepted December 30, 2015. This work was supported in part by the Research Project AGAUR under Grant 2014-SGR1551, by the Research Project CellFive under Grant TEC2014-60130-P, and by the Research Project DEFINE-5G under Grant TEC2014-60258-C2-2-R. The review of this paper was coordinated by Prof. W. Song.

A. Bousia, E. Kartsakli, and L. Alonso are with the Department of Signal Theory and Communications, Universitat Politècnica de Catalunya, 08034 Barcelona, Spain (e-mail: alexandra.bousia@tsc.upc.edu; ellik@tsc.upc.edu; luisg@tsc.upc.edu).

A. Antonopoulos and C. Verikoukis are with Telecommunications Technological Center of Catalonia, 08860 Barcelona, Spain (e-mail: aantonopoulos@ cttc.es; cveri@cttc.es).

Color versions of one or more of the figures in this paper are available online at http://ieeexplore.ieee.org.

Digital Object Identifier 10.1109/TVT.2016.2517698 to an impressive growth of data traffic during the last few 39 years. Global mobile data traffic is expected to increase nearly 40 tenfold, reaching 24.3 EB per month by 2019 [1]. Hence, 41 mobile network operators (MNOs ${ }^{1}$ ) seek to extend their in- 42 frastructure by installing more base stations (BSs). In an effort 43 to increase the capacity of their network and meet these press- 44 ing traffic demands, they also lease capacity and bandwidth 45 resources from third parties, who deploy low-powered small- 46 cell (SC) networks to provide enhanced services via traffic 47 offloading from macro BSs to SCs during peak hours [2]. For 48 instance, Nokia Networks [3] has recently built networks of 49 interconnected SCs, enabling operators to extend the coverage 50 and increase the capacity of existing macro networks. The 51 involvement of various entities of corporate nature with differ- 52 ent financial goals generates a new ecosystem with interesting 53 dynamics to be studied. To that end, in [4], the economics 54 of offloading are investigated, whereas auction theory is used 55 to model economic transactions between conflicting parties in 56 several works [5]-[7] through offloading schemes with auction- 57 based resource-allocation problems.

The dense heterogeneous networks (HetNets) imply sig- 59 nificant increase in the capital and operational expenditures 60 (CapEx and OpEx) of MNOs [8], and as a result, there is a 61 strong motivation to investigate energy-efficient solutions to 62 bring down the energy consumption and the cost of networks. 63 This goal can be accomplished through the switching off of the 64 underutilized nodes when the traffic is significantly low. Since 65 the BSs are the most power hungry and expensive components 66 of the networks [9], the research community has shifted toward 67 the investigation of BS switching-off schemes [10]-[15]. De- 68 spite their promising results, these works examine only one tier 69 of macro BSs, whereas the presence of multiple MNOs raises 70 new challenges and open issues.

The SC deployment in current HetNets can be the key to 72 implementing novel energy and cost-efficient solutions. By 73 encouraging the traffic offloading from BSs to SCs during low 74 traffic periods, when the SC resources are most likely to remain 75 unused, part of the BS infrastructure can be switched off. Given 76 that the energy consumption of the BSs is considerably higher 77 with respect to the SCs, even when the traffic load is low, such 78 solutions can yield high energy gains for the MNOs. Thus, 79 offloading can be exploited to concentrate the users to the SCs 80

\footnotetext{
${ }^{1}$ The terms "MNO" and "operator" will be used interchangeably in the remainder of this paper.
} 
81 and allow the BSs with no traffic to be switched off. However, 82 despite the potential gains of the opportunistic exploitation of 83 the capacity of SCs and the deactivation of the redundant BSs 84 during low traffic conditions, very few research works exist in 85 the literature so far.

86 An overview of the existing techniques in energy-efficient 87 network planning is presented in [16], highlighting the advan88 tages and the shortcomings of the algorithms and revealing 89 the open issues that should be further studied in the field of 90 HetNets and operators' collaboration. In the same context, in 91 [17], an auction-based offloading scheme is proposed for the BS 92 switching off, where the operators submit a bidding value, and a 93 third party's income optimization approach is followed to solve 94 the resource-allocation problem. However, only a particular 95 network configuration is considered, where it is assumed that 96 the SC capacity is sufficient to serve the traffic of the network, 97 allowing the switching off of all the BSs.

98 Despite the clear benefits of the BSs switching off via of99 floading, the presence of multiple MNOs in the same area [18] 100 would complicate the application of this scheme since the SCs 101 may not be able to fully support the network traffic. Another 102 limitation of the auction-based state-of-the-art works [5], [6], 103 [17] consists in the fact that the MNOs propose a single 104 bidding value for the requested capacity, based on the maximum 105 predictions about the traffic that they are willing to offload. 106 However, these predictions do not always correspond to the 107 real traffic values, which can be significantly lower than the 108 maximum values, leading to increased costs for the operators 109 and inefficient use of the SC capacity resources.

\section{B. Contribution}

111 In this paper, motivated by the aforementioned issues, we 112 propose a novel energy-efficient solution for dense HetNets, 113 which considers the interests of the involved parties (MNOs and 114 third party) and the time-varying traffic characteristics. More 115 specifically, we introduce an offloading mechanism, where the 116 operators lease the capacity of an SC network owned by a third 117 party, to be able to switch off their BSs and maximize their 118 energy efficiency, when the traffic demand is low. The MNOs 119 request capacity from several SCs and can only switch off their $120 \mathrm{BSs}$ if all their requests are satisfied, enabling them to offload 121 all their traffic to the SC network.

122 To that end, the allocation of the SC resources among a 123 set of competing MNOs is mathematically formulated as an 124 auction. Since the exact resource requirements of the network 125 are unknown beforehand, the MNOs employ past reports to 126 predict the maximum expected traffic load. Then, exploiting 127 the fact that, with a high probability, the actual traffic will 128 be lower than the predicted maximum (especially during low 129 traffic periods), the MNOs submit a set of bids to the SCs, 130 requesting for lower capacity resources (with respect to the 131 maximum estimated requirements). With this approach, the SC 132 resources can be more efficiently utilized, and the MNOs are 133 likely to pay a lower price for the leased capacity, taking a 134 small risk of not being able to serve all users under some 135 circumstances (i.e., when the traffic approaches the maximum 136 predictions and the leased capacity is not sufficient). Our key contribution is to study this very interesting trade-off between 137 the potential energy and financial gains of this solution and 138 the risk of not fully satisfying all the users, thus providing the 139 MNOs with the necessary insights to decide whether it is prof- 140 itable to participate in the auction, depending on their tolerance 141 to the potential loss of some users.

In addition, the conflicting interests of the involved parties 143 are also taken into consideration. On the one hand, the MNOs 144 aim to reduce their energy consumption and expenditures by 145 offloading their traffic and switching off their BS infrastructure. 146 However, to deactivate a BS, all its traffic should be offloaded 147 to the SC network (i.e., the MNO should win in all the auctions 148 involving the particular BS). On the other hand, the third 149 party wants to maximize its income by leasing the maximum 150 possible amount for resources to the MNOs. However, the 151 resource allocation policy that maximizes the third party's 152 income may not enable the operators to switch off their BSs. 153 Our proposed auction-based strategy takes into account these 154 conflicting interests to achieve a feasible, efficient, and energy- 155 saving resource-allocation scheme.

In summary, the contribution of this paper is described as 157 follows.

158

1) Bidding Strategy: We propose a novel bidding strategy, 160 where MNOs submit a set of bids (and not only one bid) 161 to the third party, requesting different capacity resources, 162 based on the predictions about their maximum traffic. The 163 diversity of bids allows more offloading opportunities, 164 which is profitable for both the MNOs and the third party. 165

2) Auction Design and Switching Off Decision: We design 166 an auction scheme that enables the efficient usage of SCs 167 resources under low traffic conditions. Our framework 168 motivates the MNOs to quantify their tolerance about 169 requesting fewer resources and form the different levels 170 of bids. We show that the proposed auction-based scheme 171 has two desirable properties: 1) truthfulness; and 2) indi- 172 vidual rationality. The mechanism is designed based on a 173 multiobjective framework, where the conflicting interests 174 of the involved parties are considered, to provide the 175 optimal solution that maximizes the economic profit of 176 both the third party and the MNOs and minimizes the 177 network energy consumption at the same time.

3) Performance Evaluation: We validate the theoretical 179 analysis of the multiobjective problem by computing 180 the Pareto front (i.e., the set of optimal) solutions and 181 assess the effectiveness of the proposed auction-based 182 switching-off algorithm. The analytical and simula- 183 tion results indicate the potential energy efficiency and 184 economic gains in the network and give the necessary 185 insights to the MNOs to decide whether it is beneficial 186 to enter in a resource allocation negotiation with the 187 third party.

The remainder of this paper is organized as follows. The 189 system model is described in Section II. In Section III, we in- 190 troduce the auction-based optimization approach that is used for 191 the BSs' switching-off decision. The performance evaluation is 192 provided in Sections IV and V concludes this paper. 


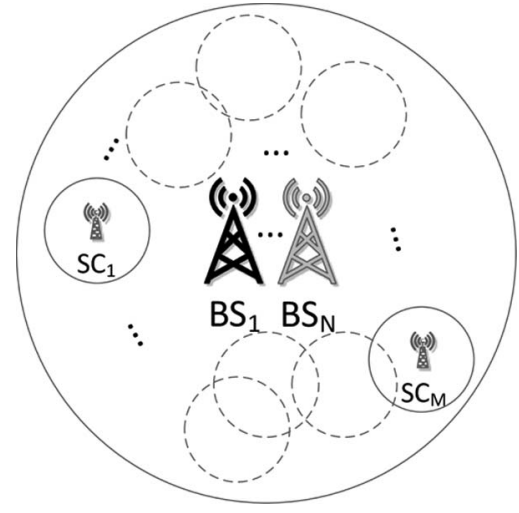

Fig. 1. Network configuration with one macro cell served by $N$ MNOs and covered by $M$ third-party SCs.

\section{SYSTEM MODEL AND OPERATION}

\section{A. Network Configuration}

196 We consider an urban scenario, focusing on an area of a 197 macro cell. In the macro cell, we assume that $N$ MNOs provide 198 coverage through their BSs, which are denoted by $\mathrm{BS}_{n}$, where $199 n \in \mathcal{N}=\{1, \ldots, N\}$ characterizes the $\mathrm{MNO}_{n}$. Let us high200 light that, in dense networks, due to legal regulations, MNOs 201 are obligated to install their antennas and BSs on the same 202 buildings; thus, we examine networks with collocated BSs [18]. 203 Moreover, in the macro cell, SCs, owned by a third party, are 204 randomly distributed. Each $\mathrm{SC}$ is represented as $\mathrm{SC}_{m}$, where $205 m \in \mathcal{M}=\{1, \ldots, M\}$. We assume that the number of SCs is 206 adequate to cover the area of the macro cell [19]. As it will be 207 explained in the following, the MNOs are motivated to offload 208 their traffic to the third-party SCs by paying the corresponding 209 price, thus enabling part of BS infrastructure to be switched 210 off during low traffic conditions. The network configuration is 211 shown in Fig. 1.

\section{B. Traffic Load Model}

213 In this paper, we adopt a realistic traffic pattern [20], [21] 214 that corresponds to the maximum traffic per operator in a given 215 cell. Fig. 2(a) plots the maximum traffic per hour, which is 216 denoted by Load $^{\max }(h)$, throughout the day. ${ }^{2}$ Without loss of 217 generality, we focus on the time zone between 01:00 A.M. and 218 09:00 A.M., when the traffic per BS is relatively low (i.e., less 219 than $40 \mathrm{Mb} / \mathrm{s}$, which corresponds to $35 \%$ of the cell's capacity). 220 The selection of the night zone may vary according to the traffic 221 variations, and our algorithm can be adapted to different traffic 222 conditions. In addition, we assume that the traffic volumes of 223 different MNOs may be different, although they follow the 224 same pattern. Hence, we define $\rho_{n} \in[0,1]$ as the percentage of 225 each operator's traffic load with respect to the maximum traffic 226 for the respective hour. In this paper, we have used traffic data 227 sets that include information of one operator for ten BSs during 228 the period of one year, thus including weekends, weekdays, 229 and day and night hours. To that end, the reports consists of 230 various values for both low and high traffic. Provided that the

\footnotetext{
${ }^{2}$ The parameter $h$ can be dropped for the sake of simplicity.
}

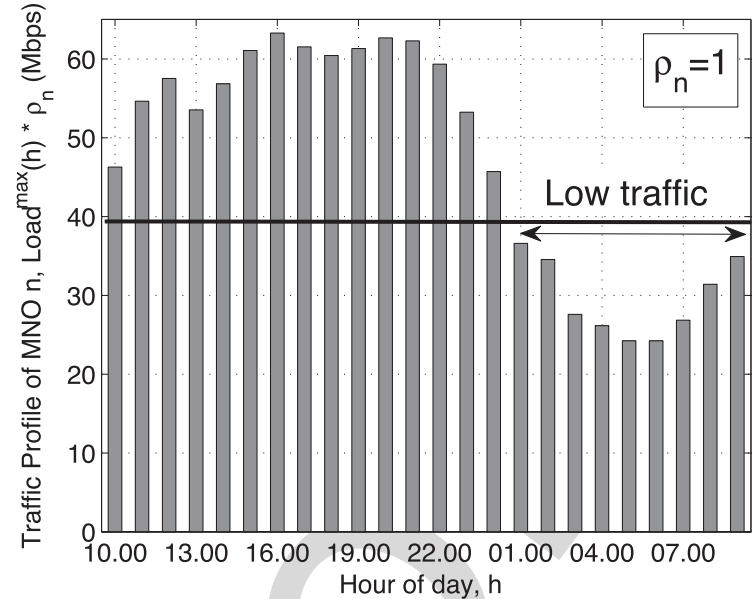

(a)

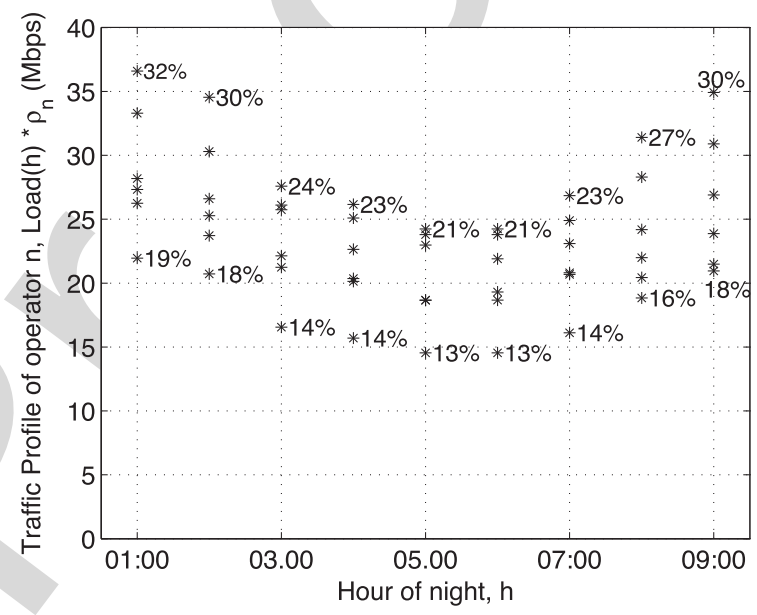

(b)

Fig. 2. Traffic pattern scenario and real data traffic values. (a) Traffic load during the 24-hour day. (b) Real data for traffic load patterns.

data sets had huge information, we classified the days of the 231 year into different periods of time (e.g., seasons, weekdays, 232 and weekends). Based on these real traffic reports and by 233 using simple statistical analysis, we have obtained the necessary 234 insights about the minimum, maximum, and average values of 235 the traffic load, and we have plotted some indicative numbers in 236 Fig. 2(b). Each one of the six values corresponds to the average 237 traffic that was observed in different days during the year. In 238 addition, we express the BS utilization as a percentage of the 239 total BS's capacity resources for the extreme cases of minimum 240 and maximum traffic loads. The two values, i.e., Load ${ }^{\mathrm{min}}$ and 241 Load $^{\text {max }}$, are very critical, and it is very important for the 242 MNOs to be able to predict them.

Unlike the existing works in the literature, where each MNO 244 places only one bid corresponding to the maximum capacity 245 requirements, in this paper, we propose that the MNOs place 246 multiple bids corresponding to different levels of the predicted 247 traffic load, as shown in Fig. 2(b). Thus, given the estimated 248 minimum and maximum traffic load levels and assuming that 249 the actual traffic values will, most probably, lie between these 250 extreme values, we are able to calculate different levels of 251 traffic. In this paper, we consider $L+1$ different traffic load 252 levels. The number of levels depends on the MNOs' strategy 253 


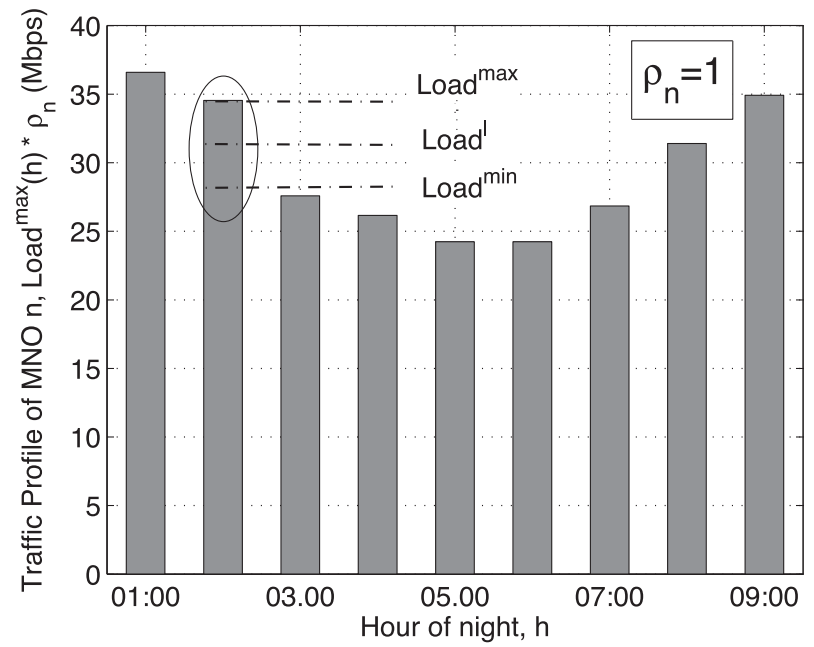

Fig. 3. Traffic during the night zone and different levels of traffic estimation.

254 that will be further explained in the following. Each level $l \in$ $255 \mathcal{L}=\{0, \ldots, L\}$ corresponds to traffic that is equal to

$$
\begin{aligned}
\operatorname{Load}^{l} & =\operatorname{Load}^{\text {min }}+\frac{\text { Load }^{\text {max }}-\text { Load }^{\text {min }}}{L} \cdot l \\
& =\left(1-\frac{l}{L}\right) \cdot \operatorname{Load}^{\text {min }}+\frac{l}{L} \cdot \operatorname{Load}^{\text {max }} .
\end{aligned}
$$

256 Evidently, the two extreme values are $\operatorname{Load}^{0}=\operatorname{Load}^{\mathrm{min}}$ and $257 \mathrm{Load}^{L}=$ Load $^{\text {max }}$. Fig. 3 shows an example, depicting various 258 traffic levels for a specific hour $(\mathrm{h}=02: 00$ A.M.). Based 259 on the above, Appendix A of the supplemental file provides 260 a theoretical estimation of the traffic that can be offloaded, 261 explaining the process of mapping the users of the MNOs to 262 the capacity of the SCs.

\section{III. Auction-Based Switching-OfF Algorithm}

264 Here, we present the combinatorial auction employed to 265 select the MNOs that can offload their traffic to the SCs, thus 266 being able to switch off their BSs. We provide the bidding strat$267 \mathrm{egy}$, and we formulate the integer linear programming model, 268 which ensures the optimal allocation and the switching-off 269 strategy for the auction. In addition, to ensure truthful bidding, 270 the Vickrey-Clarke-Grooves (VCG) payment mechanism 271 [22] is employed.

\section{A. Big Picture}

273 By considering the limited capacity of the SCs and the 274 MNOs' incentive to switch off their BSs, we use an auction275 based mechanism to motivate the operators to offload the traffic 276 to the third-party SC network. Fig. 4 illustrates the main idea of 277 the scheme. In our proposal, both the MNOs and the third party 278 take part in the decision process, each one having a separate 279 role in the framework. On one hand, the MNOs act as buyers, 280 who are willing to lease the SCs resources and opportunistically 281 offload their traffic. On the other hand, the third party, acting as 282 a seller, collects the bids, and through an auction, the subset of 283 MNOs that can offload their traffic, is selected. The proposed 284 auction-based switching-off scheme consists of three main
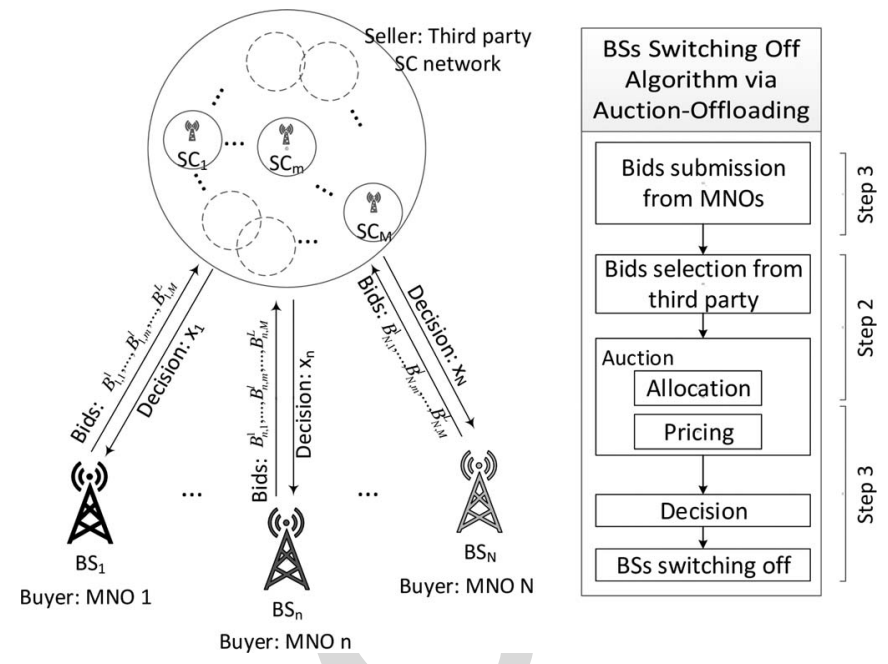

Fig. 4. Auction illustration and proposed algorithm flowchart.

steps: bidding, allocation, and pricing, whose process and the 285 respective decision-makers (in parenthesis) are presented as fol- 286 lows. First, in the bidding phase (MNOs), the MNOs place their 287 bids to the third party according to the different values of the 288 requested bandwidth. Each bid includes the information of the 289 requested capacity and the corresponding price that the MNO is 290 willing to offer. Second, in the allocation step (third party and 291 MNOs), the third party collects the MNOs' bids. The selection 292 of the optimal resource allocation can be derived through the so- 293 lution of the resource-allocation problem. Third, in the pricing 294 step (third party), the third party decides each winner's payment 295 price, based on the resource allocation of the previous step. The 296 winning MNOs offload their whole traffic to the SCs and switch 297 off their BSs, whereas the losing bidders keep their BSs active. 298

\section{B. Bidding Strategy}

Each $\mathrm{SC}_{m}$ of the third party (seller) has unexploited capacity 300 resources that is willing to lease to the MNOs. The MNOs 301 (buyers) want to offload their traffic to the SCs by requesting 302 specific capacity resources to lease, based on the predictions 303 of the traffic load. The number of the physical resource blocks 304 (PRBs) is calculated in Appendix A of the supplementary 305 file. The MNOs valuate the requested resource $N_{\mathrm{PRB}_{n, m}}^{l}$ at a 306 given price $u_{n, m}^{l}$, unknown to the third party and the other 307 bidders, where $l$ is the level of resources, $n \in \mathcal{N}$ refers to 308 the corresponding operator, and $m \in \mathcal{M}$ corresponds to the 309 specific SC that the MNO wants to lease the resources from. In 310 the proposed auction-based scheme, each operator submits a set 311 of bid pairs $B_{n, m}^{l}=\left(b_{n, m}^{l}, N_{\mathrm{PRB}_{n, m}}^{l}\right)$, representing the price 312 $b_{n, m}^{l} \leq u_{n, m}^{l}$, which the $n$th MNO pays for leasing the capacity 313 $N_{\mathrm{PRB}_{n, m}}^{l}$ from the $m$ th SC. In general, these two values (i.e., 314 $b_{n, m}^{l}, u_{n, m}^{l}$ ) may not necessarily be the same. However, in a 315 truthful auction such as the one that we have (the truthfulness 316 property of the auction will be explained in Section III-D), it 317 is proved that the private valuation and the bidding price are 318 equal; thus, $b_{n, m}^{l}=u_{n, m}^{l}$ [5], [6]. After the reception bid pairs, 319 the selection of the subset of the MNOs, whose traffic can be 320 offloaded to the corresponding SCs, follows. 


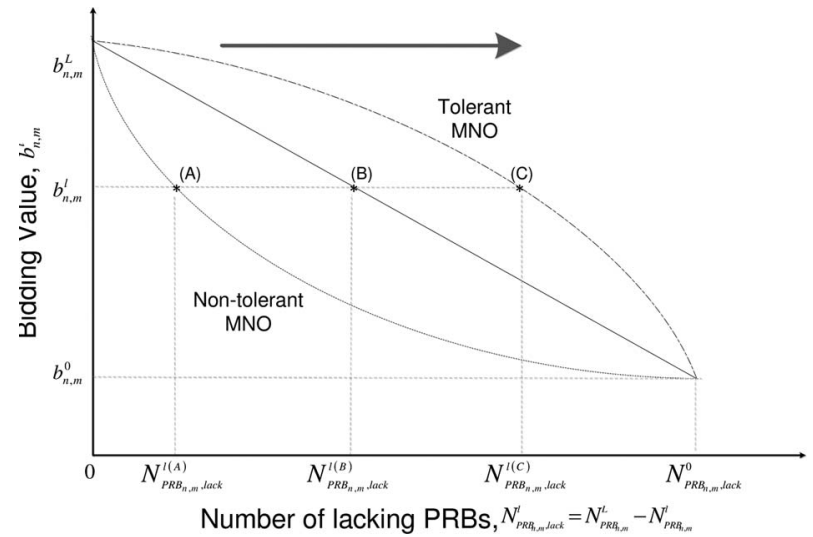

Fig. 5. Bidding strategy versus tolerance function.

322 To gain further insights on the bidding strategy, let us 323 consider the following example. Given the predicted expecta324 tions about the traffic, each MNO $m$ submits multiple bids, $325 b_{n, m}^{0}, b_{n, m}^{1}, \ldots, b_{n, m}^{l}, \ldots, b_{n, m}^{L}$, indicating the offered price for 326 the requested number of PRBs. Through the different bid pairs, 327 the MNOs actually reveal their outage probability tolerance. 328 In particular, by leasing the maximum calculated number of $329 \operatorname{PRBs}\left(N_{\mathrm{PRB}_{n, m}}^{L}\right)$ for the highest bid $b_{n, m}^{L}$, the MNOs guarantee 330 service to all their users. Controversially, by obtaining a smaller 331 number of PRBs (e.g., $N_{\mathrm{PRB}_{n, m}}^{l}$ with $l<L$ ), the MNO pays 332 a smaller price at a risk of leaving some users in outage. 333 The minimum number of requested PRBs $N_{\mathrm{PRB}_{n, m}}^{0}$ constitutes 334 an upper bound of the operator's outage tolerance. Another 335 parameter determined by each MNO is the number of levels. $336 \mathrm{~A}$ higher number of levels results in more bid pairs, thus 337 increasing the performance of the auction while inducing more 338 communication overhead and higher computational complexity. 339 To flexibly model the MNOs' outage tolerance, we introduce 340 a satisfaction function that represents the bidding value (which 341 is equal to the valuation price) that the MNO is willing to 342 pay for leasing a specific bandwidth. To that end, we define 343 the number of lacking PRBs as the difference between the 344 maximum number of PRBs minus the actually obtained PRBs 345 allocated the MNO, given by

$$
N_{\mathrm{PRB}_{n, m}, \text { lack }}^{l}=N_{\mathrm{PRB}_{n, m}}^{L}-N_{\mathrm{PRB}_{n, m}}^{l} .
$$

346 The satisfaction function is determined by the requested ca347 pacity of each MNO and is monotonically decreasing with the 348 number of lacking PRBs. We also consider that, for each MNO, 349 there is a lower bound for the minimum number of requested 350 PRBs, which minimizes the MNO's satisfaction. This bound 351 indicates that for fewer PRBs the MNO will not participate in 352 the auction. Fig. 5 shows three examples of the outage tolerance 353 function. Three bidding values are emphasized in the plot: The 354 highest bid $b_{n, m}^{L}$, corresponding to the maximum requested 355 capacity $N_{\mathrm{PRB}_{n, m}}^{L}$ (with $N_{\mathrm{PRB}_{n, m} \text {, lack }}^{l}=0$ ), the lowest bid $356 b_{n, m}^{0}$, and an intermediate value $b_{n, m}^{l}$. As the number of lacking 357 PRBs increases (depicted by the arrow direction in Fig. 5), the 358 bid values decrease, and as a consequence, losses in terms of the 359 served users may be observed. If the number of lacking PRBs 360 exceeds $N_{\mathrm{PRB}_{n, m}}^{0}$, lack , the MNO will not participate in the auc- tion; thus, no bid lower than $b_{n, m}^{0}$ will be submitted. The three 361 points (A, B, and C) marked in Fig. 5 correspond to the different 362 tolerance functions of three operators. An outage-tolerant MNO 363 (point $\mathrm{C}$ ) requests for less bandwidth for the same bidding value 364 $b_{n, m}^{l}$ compared with a nontolerant MNO (point A) that still 365 requests high capacity from the third party. Hence, the nontol- 366 erant MNO places higher priority on guaranteeing user service, 367 whereas the outage-tolerant MNO is willing to sacrifice some 368 resources to increase its probability of winning the auction and 369 switching off its BS, thus enhancing energy efficiency. Finally, 370 point $B$ corresponds to an intermediate bidding strategy defined 371 by a linear function between the two examined parameters. $\quad 372$

The user outage tolerance function (keeping in mind that 373 $\left.b_{n, m}^{l}=u_{n, m}^{l}\right)$ is modeled as $^{3}$

$$
b_{n, m}^{l}=b_{n, m}^{L}-\frac{b_{n, m}^{L}-b_{n, m}^{0}}{N_{\mathrm{PRB}_{n, m}}^{L}-N_{\mathrm{PRB}_{n, m}}^{0}} \cdot\left(N_{\mathrm{PRB}_{n, m}}^{l}\right)^{\delta} .
$$

The distinctive cases for the satisfaction curves are as 375 follows.

- Nontolerant MNO (Point A): For the nontolerant MNO, 378 the outage tolerance function is convex, with $\delta<1$. 379

- Average-tolerant MNO (Point B). There is a linear re- 380 lation between the bidding strategies and the outage 381 tolerance of the MNOs, by substituting $\delta=1$ in (3). $\quad 382$

- Tolerant MNO (Point C). For the tolerant MNO, the 383 satisfaction function is concave, with $\delta>1$.

The parameter $\delta$ may obtain a wide range of values. The selec- 385 tion of a value equal to $\delta \ll 1$ corresponds to a strictly nontoler- 386 ant MNO, who decreases its bid requests very fast. In contrast, a 387 very high value of this indicative parameter (i.e., $\delta \gg 1$ ) would 388 lead to very slowly decreasing bidding values and, thus, to a 389 very tolerant operator.

\section{Auction Formulation}

By employing the properties of combinatorial auction theory, 392 we formulate the problem of the opportunistic offloading as 393 an auction. Each MNO $n \in \mathcal{N}$ places the corresponding bid 394 pairs $B_{n, m}^{l}$. Having received the bids, the third party selects 395 the subset of MNOs that maximizes the desired goals of all the 396 involved participants. We define $x_{n, m}^{l}$ as a binary decision vari- 397 able that indicates whether the corresponding bidder (MNO $n$ ) 398 is winner $\left(x_{n, m}^{l}=1\right)$ or not $\left(x_{n, m}^{l}=0\right)$ in the corresponding 399 $\mathrm{SC}_{m}$ and for the $l$ th bidding value.

400

The cost of using the SC infrastructure and the increased 401 consumed energy are the main objectives: the maximization 402 of the profits (the economic objectives of the third party and 403 the MNOs) and the minimization of the energy consumption 404 (the energy objective). In continuation, a detailed analysis of 405 the objectives of each party is presented.

406

1) Third Party's Objective: The third party aims at maximiz- 407 ing its profit by leasing high volumes of the capacity of SCs at 408

\footnotetext{
${ }^{3}$ The values of $b_{n, m}^{0}$ and $b_{n, m}^{L}$ will be calculated in details in the succeeding section, where the constraints and requirements posed by the MNOs and the third party are given within the optimization framework.
} 
409 increased prices. The profit is defined as the difference of the 410 MNOs' winning bids minus its expenses (CapEx and OpEx). 411 We define the financial gain of the $\mathrm{SC}$ network $\mathrm{CG}_{\mathrm{SC}}$ as

$$
f_{1}(\mathbf{x})=\mathrm{CG}_{\mathrm{SC}}=\sum_{n \in \mathcal{N}} \sum_{m \in \mathcal{M}} \sum_{l \in \mathcal{L}} x_{n, m}^{l} \cdot b_{n, m}^{l}-M \cdot C_{\mathrm{SC}}
$$

412 where $C_{\mathrm{SC}}$ is the cost of a SC, corresponding to the sum of its $413 \mathrm{CapEx}$ and the traffic-dependent OpEx. For the calculation of 414 the SCs cost, the cost model presented in [23] is employed.

415 To ensure its objective, the third party introduces a minimum 416 profit, which is described as a function of its total cost, i.e.,

$$
\mathrm{CG}_{\mathrm{SC}}^{\min }=y \cdot M \cdot C_{\mathrm{SC}}, \quad y>0
$$

417 Given the minimum profit, which is calculated by (5), we 418 estimate the reservation price as follows.

419 Proposition 1: The reservation price is defined as the mini420 mum price that a seller would be willing to accept for leasing 421 the corresponding bandwidth, i.e.,

$$
b_{\mathrm{res}, n, m}^{l}=\frac{N_{\mathrm{PRB}_{n, m}}^{l}}{N_{\mathrm{PRB}}^{m a x}} \cdot y \cdot M \cdot C_{\mathrm{SC}} .
$$

422 Proof: The third party is willing to share its resources 423 among the MNOs based on proportional fairness by ensuring 424 that a minimum profit gain will be achieved, at the same time. 425 To that end, it calculates a reservation price, which is the 426 minimum price that the third party will accept from an MNO 427 to lease a specific bandwidth. The maximum number of PRBs 428 that the $n$th MNO can offload to the $m$ th SC is given by

$$
N_{\mathrm{PRB}_{n, m}}^{l}=\frac{b_{\mathrm{res}, n, m}^{l} \cdot x_{n, m}^{l}}{\sum_{n \in \mathcal{N}} \sum_{l \in \mathcal{L}} b_{\mathrm{res}, n, m}^{l} \cdot x_{n, m}^{l}} \cdot N_{\mathrm{PRB}}^{\mathrm{max}}
$$

429 Finally, by using (5) and (7) in (4), the reservation price for 430 offloading traffic is calculated.

431 The reservation price in (6) is not the price that the MNOs 432 will propose to lease their requested capacity, although it is 433 a threshold price that the third party uses to eliminate all the 434 offers that are less than the accepted. The reservation price can 435 be either announced or unknown to the MNOs. The reservation 436 price means that the seller would rather withhold the capacity 437 if the proposed bids are too low (i.e., lower than the reservation 438 price), and given this price, the auction process can be acceler439 ated since the set of prices that are lower than the reservation 440 price can be discarded.

441 2) MNOs' Objective: The objective of each MNO is the 442 maximization of its financial profits. The maximization of the 443 profits of the $n$th MNO is defined as the revenue from switching 444 off its BS minus the winning bids for leasing the requested 445 capacity from the third party. Therefore, the profit (cost gain) 446 can be written as

$$
f_{2}(\mathbf{x})=\mathrm{CG}_{n}=C_{\mathrm{BS}} \cdot x_{n}-\sum_{m \in \mathcal{M}} \sum_{l \in \mathcal{L}} x_{n, m}^{l} \cdot b_{n, m}^{l}
$$

with

$$
x_{n}=\prod_{m \in \mathcal{M}} \sum_{l \in \mathcal{L}} x_{n, m}^{l} \quad \forall n \in \mathcal{N}
$$

and $C_{\mathrm{BS}}$ being the cost of a $\mathrm{BS}$, whose model is also presented 448 in [23], provided that

$$
\sum_{l \in \mathcal{L}} x_{n, m}^{l} \in\{0,1\} \quad \forall n \in \mathcal{N} \quad \forall l \in \mathcal{L}
$$

At this point, let us recall that an operator is able to switch 450 off its BS if it wins in an auction in all the SCs, a condition that 451 is represented by the product in (9). The product is equal to 1452 only when the $n$th MNO wins in $M$ auctions in respective SCs; 453 otherwise, it is 0 .

The MNOs are willing to participate in the auction and lease 455 bandwidth resources from a third party if they guarantee a mini- 456 mum profit gain. Consequently, they introduce a minimum pro- 457 fit, which is described as a percentage of their total costs, i.e., $\quad 458$

$$
\mathrm{CG}_{n}^{\min }=z \cdot C_{\mathrm{BS}}, \quad 0<z<1 .
$$

The bidding strategy of the MNOs, along with the proposed 459 bids, depend on the minimum profit gain and the operation costs 460 of the BSs. However, the cost gain can be attained if and only 461 if the $n$th MNO wins in $M$ auctions and is able to lease the 462 requested capacity. In addition, since we consider uniform traf- 463 fic in the macro cell, we conclude that the bids in the different 464 SCs must be equal and proportional to the corresponding traffic. 465 Thus, the maximum bid price for offloading the traffic in the 466 $m$ th cell is calculated as

$$
b_{n, m}^{L}=\frac{(1-z) \cdot C_{\mathrm{BS}}}{M} .
$$

Similarly, the minimum bidding price is a proportional value 468 of the maximum one, which is given by

$$
b_{n, m}^{0}=v \cdot \frac{(1-z) \cdot C_{\mathrm{BS}}}{M}
$$

where $v \in(0,1)$. The bidding values of the remaining $L-1470$ levels can be calculated based on (3), depending on the maxi- 471 mum bid value and the relation between the outage tolerance of 472 the MNOs and the discount they can be offered for the lower 473 requested bandwidth.

3) Overall Objective: The overall objective of the network 475 is the minimization of the energy consumption. This can be 476 attained by reducing the number of active BSs, given that the 477 BSs are responsible for the major part of energy consumption 478 in the network. The network energy consumption $\mathbb{E}[E]$ is

479

$f_{3}(\mathbf{x})=\mathbb{E}[E]=\sum_{n \in \mathcal{N}} \mathbb{E}\left[E_{\mathrm{BS}_{n}}\right] \cdot\left(1-x_{n}\right)+M \cdot \mathbb{E}\left[E_{\mathrm{SC}}\right]$

where $\mathbb{E}\left[E_{\mathrm{BS}_{n}}\right]$ and $\mathbb{E}\left[E_{\mathrm{SC}}\right]$ represent the energy consumption 480 of $\mathrm{BS}$ and $\mathrm{SC}$, respectively. 
482 The combinatorial auction formulation should include all the 483 objectives of the participating entities. To capture the trade-off 484 between the objectives, the multiobjective optimization [24] is 485 employed. The target is to find a subset of acceptable solutions 486 according to a set of objectives. In general terms, the objectives 487 may be conflicting, and consequently, a single global optimum 488 may not exist. Hence, the notion of an optimum set $\mathrm{x}^{*}$ becomes 489 very important. Some relevant definitions are given in the 490 following.

491 Definition 1: Given the three objectives, $f_{1}(\mathbf{x}), f_{2}(\mathbf{x})$, and $492 f_{3}(\mathbf{x})$, and provided that $\mathbf{x}_{1}$ and $\mathbf{x}_{2}$ are two decision variables, 493 then $\mathbf{x}_{1}$ is said to be the Pareto dominant, and is denoted $\mathbf{x}_{1} \succeq$ $494 \mathbf{x}_{2}$ if and only if $f_{i}\left(\mathbf{x}_{1}\right) \geq f_{i}\left(\mathbf{x}_{2}\right) \forall i \in\{1,2,3\}$, and $f_{j}\left(\mathbf{x}_{1}\right)>$ $495 f_{j}\left(\mathbf{x}_{2}\right)$, for at least one index $j \in\{1,2,3\}$.

496 Definition 2: $\mathrm{x}^{*}$ is said to be Pareto optimal (or nondom497 inated), if there is no other $\mathbf{x}$, so that $\mathbf{x}$ dominates $\mathbf{x}^{*}$. The 498 set of all Pareto solutions in the decision space is called the 499 Pareto optimal set and the image of the Pareto optimal set in 500 the objective space is called the Pareto optimal front.

AQ3 501 Based on the aforementioned definitions, the ILP multiobjec502 tive optimization problem can be formulated as follows.

503 Definition 3: The allocation problem is to determine the 504 optimal solution $\left\{x_{n, m}^{l}\right\} \forall n \in \mathcal{N} \forall m \in \mathcal{M}$, and $\forall l \in \mathcal{L}$ that 505 maximizes the distinctive objectives of the involved parties in 506 the auction, subject to capacity and offloading targets

P1 : $\max \left[\mathrm{CG}_{\mathrm{SC}}, \mathrm{CG}_{n},-\mathbb{E}[E]\right]$

s.t.

$$
\begin{aligned}
& \sum_{n \in \mathcal{N}} \sum_{l \in \mathcal{L}} x_{n, m}^{l} \cdot N_{\mathrm{PRB}_{n, m}}^{l} \leq N_{\mathrm{PRB}}^{\max } \forall m \in \mathcal{M} \\
& \sum_{l \in \mathcal{L}} x_{n, m}^{l} \in\{0,1\} \quad \forall n \in \mathcal{N} \quad \forall m \in \mathcal{M} \\
& b_{n, m}^{l} \geq b_{\mathrm{res}, n, m}^{l} \forall n \in \mathcal{N} \forall m \in \mathcal{M} \forall l \in \mathcal{L} \\
& x_{n, m}^{l} \in\{0,1\} \quad \forall n \in \mathcal{N} \quad \forall m \in \mathcal{M} \quad \forall l \in \mathcal{L} .
\end{aligned}
$$

507 The constraint in (16) ensures that the total number of 508 allocated resources does not exceed their availability, constraint 509 (17) ensures that only one bid of the $n$th MNO in the $m$ th SC 510 can be the winning bid among the $l$ different ones, constraint 511 (18) ensures that the bids are higher than the reservation price, 512 and constraint (19) ensures the integrality of the binary variable. 513 The objectives of the proposed optimization formulation are 514 contradictory. The maximization of the third party income does 515 not imply the BSs switching off, whereas the energy objective 516 does not ensure the third party's interests. The maximization 517 of the financial gains of the third party may lead to additional 518 economic losses from the MNOs' perspective since the maxi519 mization of this objective may lead to a resource allocation that 520 does not imply the deactivation of BSs.

521 Exploiting the fact that the BSs are responsible for the major 522 part of the energy consumption, the third objective of the prob523 lem $f_{3}(\mathbf{x})$ can be transformed into a constraint. Energy con524 sumption is minimized when the maximum number of MNOs 525 switches off their BSs after winning in $M$ auctions, a condition 526 represented by the product in (9). Thus, the problem P1 is transformed into a simpler formulation in (20), and at the same 527 time, the energy consumption objective is not neglected, in 528 contrast to former works [17], where only the maximization of 529 the third party's income is considered. The equivalent problem 530 is as follows:

P2: $\max \left[\mathrm{CG}_{\mathrm{SC}}, \mathrm{CG}_{n}\right]$

s.t.

$$
\begin{aligned}
& \prod_{m \in \mathcal{M}} \sum_{l \in \mathcal{L}} x_{n, m}^{l} \in\{0,1\} \quad \forall n \in \mathcal{N} \\
& \sum_{n \in \mathcal{N}} \sum_{l \in \mathcal{L}} x_{n, m}^{l} \cdot N_{\mathrm{PRB}_{n, m}}^{l} \leq N_{\mathrm{PRB}}^{\max } \forall m \in \mathcal{M} \\
& \sum_{l \in \mathcal{L}} x_{n, m}^{l} \in\{0,1\} \quad \forall n \in \mathcal{N} \quad \forall m \in \mathcal{M} \\
& b_{n, m}^{l} \geq b_{\mathrm{res}, n, m}^{l} \forall n \in \mathcal{N} \quad \forall m \in \mathcal{M} \quad \forall l \in \mathcal{L} \\
& x_{n, m}^{l} \in\{0,1\} \quad \forall n \in \mathcal{N} \forall m \in \mathcal{M} \quad \forall l \in \mathcal{L} .
\end{aligned}
$$

The objective function (20) aims at maximizing the financial 532 gain of both the third party and the MNOs. Constraint (21) 533 ensures that an operator either wins in one auction in the $M 534$ SCs (and switches off its BS) or loses in all the auctions (keeps 535 its BS active). Thus, this constraint ensures the minimization 536 of the energy consumption given by (14) since the selected 537 resource allocation leads to the highest number of switched-off 538 BSs, while at the same time achieving the increase in MNOs 539 and third party's income. Constraints (22)-(25) are the same as 540 (16)-(19), respectively.

541

The multiobjective problem $\mathbf{P 2}$ belongs to the class 542 NP-Complete since it is equivalent to the 0-1 knapsack prob- 543 lem, which is a well-known problem in combinatorial optimiza- 544 tion. For the NP-hard problems, an optimal solution is difficult 545 to be found due to the large number of variables [24]. However, 546 there are different solutions that represent the best feasible state 547 of the investigated system, e.g., the best resource allocation 548 for the deactivation of the highest number of BSs. To find the 549 best possible representation or a good approximation of these 550 optimal solutions that are widely known as the Pareto optimal 551 set or as the optimal Pareto front and to overcome the high 552 complexity of multiobjective problems, metaheuristic methods 553 (also sometimes called genetics) have become a very active 554 research area, and several algorithms have been proposed [25], 555 [26]. Although, the metaheuristic algorithms do not guarantee 556 that this approximation is the optimal, the solutions are still 557 good and near optimal. ${ }^{4}$

The metaheuristic algorithm that finds the Pareto solution 559 for our multiobjective optimization problem works on a set 560 of possible combinations of the decision variables [24]. The 561 basic definition of the Pareto front is that it consists of exactly 562 those point solutions that are not dominated by any other point. 563 The different objectives cannot be optimized simultaneously. 564 Thus, first, the solutions that maximize the MNOs's income 565 and the third party's economic gains are calculated separately. 566

\footnotetext{
${ }^{4}$ For the sake of simplicity, we use the term "Pareto front" throughout this paper to refer to the obtained solutions through metaheuristics.
} 
567 Obviously, these solutions that maximize the cost gains of the 568 MNOs and the third-party always belong to the Pareto front 569 and, in fact, they are its endpoints. A simple algorithm to 570 find the other solutions (if any) on the Pareto front begins by 571 sorting the solutions according to one of the objectives, e.g., 572 third party's income. The algorithm then starts with the point 573 with the maximum gain for the third party and continues to 574 the successive solutions in order of increasing third party's cost 575 until the solutions with higher cost gain value for the operators 576 is found. This solution is then added to the Pareto front, and the 577 search is restarted from it.

578 Through the use of metaheuristics, the problem can be solved 579 efficiently and quickly for a relatively small number of MNOs 580 and SCs [25], [27], [28]. This assertion cannot be concluded 581 and generalized for large networks. In the investigated network 582 configuration with $5 \mathrm{MNOs,} 15 \mathrm{SCs}$, and 9 bidding levels, the 583 runtime of the solution is very short. At this point, let us clarify 584 that the numbers selected for the MNOs and the SCs are based 585 on the most typical and realistic scenarios in recent studies [18], 586 [19] since the most common scenarios in European countries 587 involve three to four MNOs, and 15 SCs can cover the service 588 are of one macro BS.

\section{D. Pricing Strategy}

590 Having defined the ILP model, we now illustrate the payment 591 rule, which is of crucial importance for the realization of the 592 auction for the third party. The VCG payment induces all 593 the users to reveal their actual valuations for the requested 594 bandwidth. In the VCG mechanism, the third party charges the 595 bidders (MNOs) with a price for the requested capacity. For 596 example, an MNO who requires large bandwidth will have to 597 pay a high price since its request results in dissatisfying other 598 MNOs who lose in the auction because of the limited capacity 599 resources and will not be able to offload their traffic.

600 Let us denote by $p_{n, m}^{l}$ the price paid by the MNO $n$ to the 601 third party for the allocated capacity of the $m$ th SC. The payoff 602 function for bidder $n$ that represents the difference between the 603 gain of the MNO attained through the BS switching off and 604 the true valuation minus the paid price (let us recall that in the 605 truthful auction $\left.u_{n, m}^{l}=b_{n, m}^{l}\right)$ is

$$
\mathcal{U}_{i}= \begin{cases}\mathrm{CG}_{i}+\sum_{m \in \mathcal{M}} \sum_{l \in \mathcal{L}} u_{i, m}^{l}-\sum_{m \in \mathcal{M}} \sum_{l \in \mathcal{L}} p_{i, m}^{l}, & \text { if } i \text { is selected } \\ -C_{\mathrm{BS}}, & \text { otherwise. }\end{cases}
$$

The payment rule for each MNO can be defined as follows:

$$
\begin{aligned}
p_{i, m}^{l}= & \sum_{n \in \mathcal{N} \backslash\{i\}} \sum_{m \in \mathcal{M}} \sum_{l \in \mathcal{L}}\left(x_{i, m}^{l}\right)^{\{-i\}} \cdot b_{i, m}^{l} \\
& -\left(\sum_{n \in \mathcal{N} \backslash\{i\}} \sum_{m \in \mathcal{M}} \sum_{l \in \mathcal{L}} x_{i, m}^{l} \cdot b_{i, m}^{l}\right. \\
& \left.-\sum_{n \in \mathcal{N} \backslash\{i\}} \sum_{m \in \mathcal{M}} \sum_{l \in \mathcal{L}} x_{i, m}^{l} \cdot b_{i, m}^{l}\right)
\end{aligned}
$$

TABLE I

SimUlation PARAMETERS

\begin{tabular}{rl|rl}
\hline Parameter & Value & Parameter & Value \\
\hline \hline Number of MNOs, $N$ & 5 & BS bandwidth, $C R_{B S}$ & $20 \mathrm{MHz}$ \\
Number of SCs, $M$ & 15 & SC bandwidth, $C R_{S C}$ & $5 \mathrm{MHz}$ \\
Traffic load, Load & Fig. 3 & $\delta$ & {$[0.5,1.5]$} \\
\hline
\end{tabular}

where the first term is the aggregate valuation of the allocated 607 resources, $\left(x_{n, m}^{l}\right)^{\{-i\}}$, when MNO $i$ does not participate at all 608 in the auction. The second term is the aggregate valuation of the 609 allocation $x_{n, m}^{l}$ of all MNOs other than $i$, when $i$ participates 610 in the auction. Next, we prove that our optimized auction 611 mechanism possesses two important properties: 1) truthfulness 612 and 2) individual rationality.

613

Proposition 2 (Truthfulness): The payment rule defined in 614 (27) satisfies the truthfulness property.

615

Proof: The proof is given in Appendix B in the supple- 616 mental file.

Proposition 3 (Individual Rationality): The payment rule 618 defined in (27) satisfies the individual rationality property, and 619 all bidders are guaranteed to obtain nonnegative utility.

Proof: The proof is given in Appendix C in the supple- 621 mental file.

622

\section{PERformance Evaluation}

This section is composed of three parts. First, we explain 624 the simulation scenario employed both for the multiobjective 625 optimization and the system-level simulations. Second, we 626 present the results regarding the estimation of the Pareto front 627 for the multiobjective optimization solved by using the Matlab 628 tools. Finally, we show a performance comparison between 629 the proposed scheme and two state-of-the-art schemes with the 630 development of a custom-made $\mathrm{C}$ simulator.

\section{A. Simulation Scenario}

The simulation scenario corresponds to a dense HetNet de- 633 ployment, such as a university campus or an urban area. More 634 specifically, our scenario focuses on a cell served by the BSs of 635 $N=5$ MNOs and $M=15$ SCs that cover the whole cell area. 636 In our experiments, we consider various scenarios, wherein the 637 MNOs have different traffic volumes (i.e., $\rho$ ), different bidding 638 strategies (i.e., nontolerant, linear, and tolerant), different cost 639 requirements (i.e., $z$ ), and different bidding levels (i.e., L). 640 The system-level simulations are based on Monte Carlo exper- 641 iments, and the presented results focus on the night zone for 642 the duration of one year. ${ }^{5}$ The set of parameters is provided 643 in Table I.

644

To assess the performance of our scheme, we compare 645 the proposed multiobjective auction-base switching-off strat- 646 egy (referred to as MAS), to two benchmark solutions: 1) an 647 auction-based switching-off scheme, wherein the income of the 648

\footnotetext{
${ }^{5}$ Note that the results are calculated for the case of one macro cell, but they can be easily extended to extended configurations. Furthermore, we consider the period of one year since the yearly traffic is considered stable [30].
} 


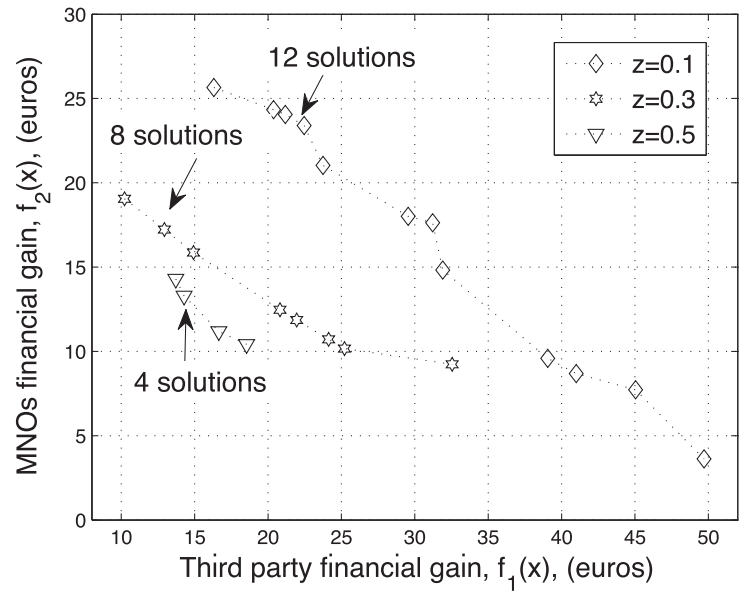

(a)

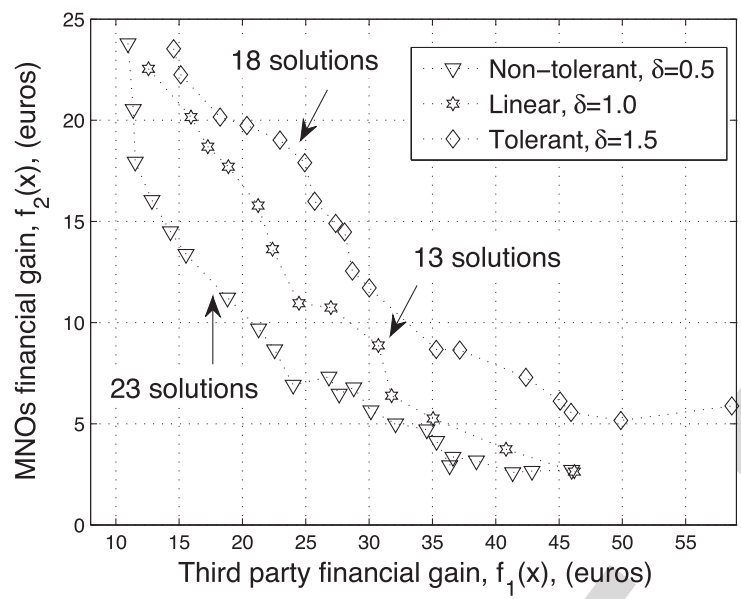

(b)

Fig. 6. Estimation of Pareto front solutions. (a) Estimated Pareto front for multiple MNOs' requirements, $L=2$ and linear bidding strategy. (b) Estimated Pareto front for multiple bidding strategies, $L=2$, and $z=0.1$.

649 third party is the unique objective to be maximized (referred 650 as ISO) [17]; and 2) a baseline scenario with full operational 651 topology (FOT), where none of the BSs is switched off.

\section{B. Estimation of the Pareto Front}

653 Here, we present the Pareto front analysis. In this set of 654 experiments, we assume that all operators have the same traffic 655 volume equal to the maximum possible traffic load, i.e., $\rho_{n}=1$. 656 In addition, we assume that each MNO submits three different 657 bids, corresponding to $L=2$, to the third party.

658 Fig. 6 shows the Pareto front solutions achieved by solving 659 the proposed multiobjective problem for different minimum 660 financial gains with the same bidding strategy [see Fig. 6(a)] 661 and different bidding strategies with the same minimum profit 662 requirement [see Fig. 6(b)]. In both figures, the third party's 663 annual financial gain that can be attained by leasing its re664 sources to one MNO is represented in the $x$-axis, and the annual 665 economic profits of one MNO are given in $y$-axis.

666 In Fig. 6(a), the set of optimal solutions is shown for the 667 linear bidding strategy. Note that the stochastic search per668 formed by means of the metaheuristic algorithm succeeds in estimating a Pareto front for the given parameters of the studied 669 problem. As expected, in all the different cases, the higher the 670 third party's income $\left(f_{1}(\mathbf{x})\right)$, the lower the financial gain of 671 the MNOs $\left(f_{2}(\mathbf{x})\right)$. Thus, the Pareto front is monotonically 672 decreasing, meaning that the improvement of one objective 673 leads to the deterioration of the other objective, and there is 674 no feasible global solution maximizing all the objectives. A 675 second observation relies on the motivation of the MNOs to 676 maximize their economic gains, which is a fact that can be 677 explored through the parameter $z$ that reflects the requirements 678 of the minimum profit gain, denoted in (11). As it is plotted 679 in the figure, the greedy behavior of the MNOs for higher cost 680 gains $(z=0.5)$ leads to lower income for the third party and, 681 at the same time, a lower number of achievable solutions. This 682 result is of significant importance since a greedy behavior from 683 the MNOs' perspective will produce lower bids that may not be 684 accepted by the third party; thus, the MNOs will not be able to 685 offload their whole traffic. The selection of a proper value of the 686 parameter $z$ helps the operators to decide whether it is profitable 687 for them to bid by taking into account their financial needs, 688 and by observing Fig. 6(a), we conclude that lower values of 689 $z$ lead to higher income for the two involved parties. To provide 690 further insights for our approach, let us also highlight that, in 691 a realistic scenario with a large number of macro BSs, the cost 692 gains for the MNOs and the third party are significantly higher. 693 For example, by applying the proposed switching off technique 694 to the central area of London where an MNO may have up to 695 500 deployed BSs [31], the economic gains may reach up to 696 $\sim 12.500 €$ for the MNO and up to $\sim 25.000 €$ for the third 697 party.

In continuation, Fig. 6(b) illustrates the Pareto front by 699 examining different bidding strategies for the case of $z=0.1 .700$ Again, we observe that the attained solutions are decreasing 701 monotonically. Moreover, as we see, the more tolerant the 702 bidding strategy of the MNOs, the higher financial gains for the 703 involved parties. This important insight can be easily explained 704 by taking into account the fact that a nontolerant MNO is more 705 greedy; thus, it requests more capacity for the same bids with 706 respect to a tolerant MNO, as shown in Fig. 5. As a result, 707 the third party may lose its incentive to lease the bandwidth, 708 and the economic benefits will be reduced for the MNOs and 709 the SC network. It is noted that the tolerant bidding strategy 710 offers better solutions due to the leasing of more resources and 711 the switching off of higher number of BSs, whereas the linear 712 bidding strategy implies an intermediate solution between the 713 extreme cases. Hence, along with the minimum guaranteed 714 profit, the bidding strategy is also an important indicator that 715 affects the decision of MNOs on bidding.

\section{Numerical Results}

Here, the performance results with regard to 718 telecommunication-oriented (network throughput and energy 719 efficiency) and cost-oriented (annual cost and income gains) 720 metrics are shown. In this set of experiments, we study a more 721 realistic scenario, where the MNOs have different traffic vol- 722 umes and, thus, different requirements and outcomes, when 723 our proposed algorithm is applied. According to recent studies 724 


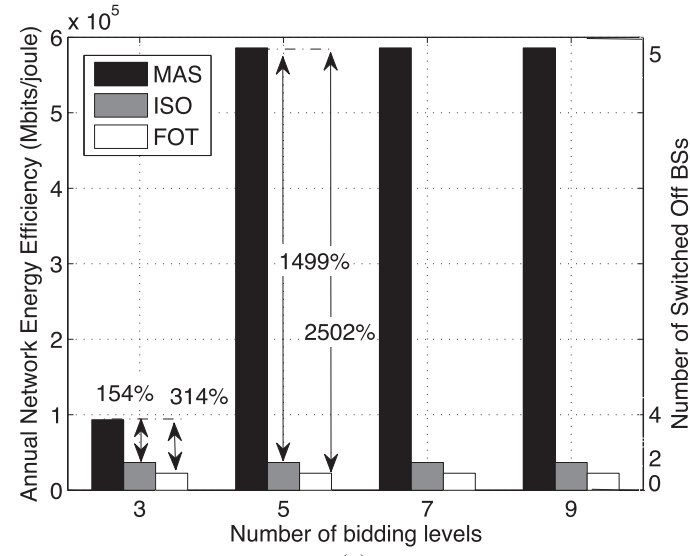

(a)

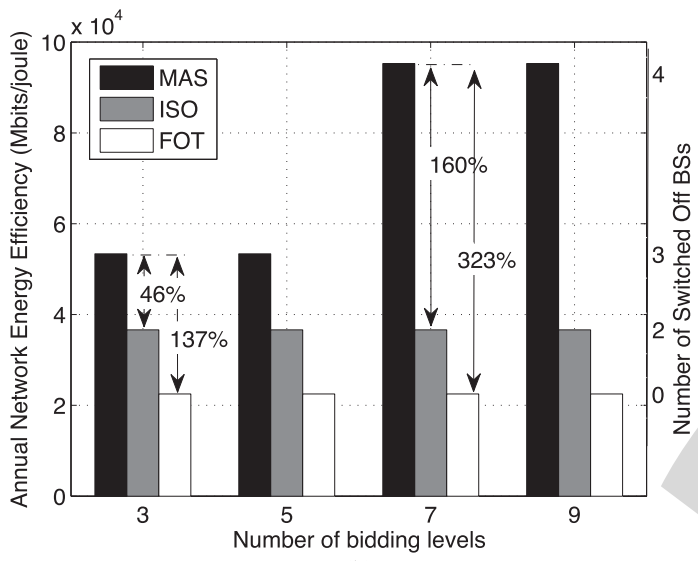

(b)

Fig. 7. Annual energy efficiency for $z=0.1$ and different bidding strategies (a) Tolerant bidding strategy. (b) Nontolerant bidding strategy.

725 [18], in most European countries, there are usually up to 726 two telecommunication companies holding the major part 727 of the market share, up to two smaller operators serving an 728 intermediate portion of the users, and finally, up to one smaller 729 MNO with a very small slice of the market. By exploiting these 730 interesting findings, we consider the scenario in which two 731 MNOs (i.e., $\mathrm{BS}_{1}$ and $\left.\mathrm{BS}_{2}\right)$ are fully loaded $\left(\rho_{1}=\rho_{2}=1.0\right.$ ), 732 two operators (i.e., $\mathrm{BS}_{3}$ and $\mathrm{BS}_{4}$ ) have relatively lower traffic 733 with $\rho_{3}=\rho_{4}=0.7$, and the fifth $\mathrm{MNO}$ (denoted by $\mathrm{BS}_{5}$ ) 734 has very low traffic $\left(\rho_{5}=0.3\right)$. The results presented in the 735 following are calculated for an operating point on the Pareto 736 front that is assumed to satisfy all the involved parties in the 737 auction. We would like to highlight that the selection of a 738 solution among the Pareto front solutions can be either derived 739 through agreements between the involved parties or another 740 distinguished entity may select it.

741 1) Telecommunication Metrics: Fig. 7 presents the total 742 network energy efficiency versus $L$ for tolerant [see Fig. 7(a)] 743 and nontolerant [see Fig. 7(b)] bidding strategies. The number 744 of switched-off BSs of every algorithm is also shown in the 745 plot (right $y$-axis), along with the percentage gains in terms 746 of energy efficiency of our approach compared with the state747 of-the-art works. First, we observe that the energy efficiency 748 achieved by the MAS scheme increases with the number of 749 levels for tolerant and nontolerant bidding since the biggest

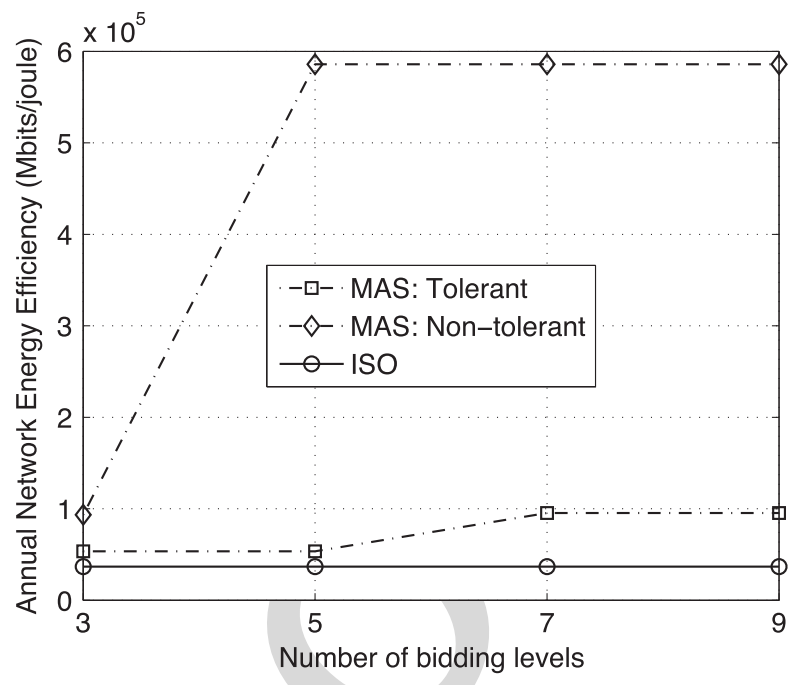

Fig. 8. Annual energy efficiency for different bidding levels and strategies.

variety of the proposed bids (higher number of levels) implies 750 a higher number of choices for the resource allocation. Another 751 important remark is that MAS significantly outperforms the 752 baseline scenario, FOT, (where no BS is switched off), and 753 the ISO algorithm. The better performance of our proposal in 754 terms of energy efficiency is justified by the higher number 755 of switched-off BSs as this is highlighted also in the plots. 756 As we have already mentioned in Section III-C, the goal of 757 our proposal lies in maximizing the energy efficiency, an ob- 758 jective neglected in the auction-based works of the literature, 759 whereby only the maximization of the third party's income 760 was considered. Comparing the behavior of our algorithm in 761 the two figures, it can be observed that the tolerant bidding 762 strategy achieves more considerable gains with respect to the 763 nontolerant bidding, mainly due to the deactivation of many 764 underutilized BSs in the network. These results emphasize 765 the role of the bidding strategy and the number of bidding 766 levels on the achievable energy efficiency, thus providing the 767 necessary insights to the MNOs to select the most appropriate 768 strategy based on their interests. In Fig. 8, the conclusions of 769 Fig. 7(b) and (a) are better illustrated since the comparison 770 between the three schemes and the different bidding strategies 771 is more clearly shown. Finally, we should mention that the 772 results of linear bidding are not shown here for simplicity, given 773 that the achieved gains range between the two aforementioned 774 cases.

Along with the total network energy efficiency performance, 776 it is interesting to study the individual energy efficiency gains 777 of the different MNOs. To that end, the individual gains for 778 the specific (but representative) case of $z=0.1$ and the two 779 different bidding strategies are quantified in Table II, where 780 interesting conclusions can be extracted. In particular, inde- 781 pendently of the bidding strategy and the number of $L$, the 782 ISO scheme is beneficial only for the group of operators that 783 switch off their BSs, who are always the same $\left(\mathrm{MNO}_{1}\right.$ and 784 $\mathrm{MNO}_{2}$ ), whereas the rest of the operators always keep their 785 BSs active. More specifically, the MNOs that switch off their 786 BSs theoretically achieve infinite energy efficiency, as they 787 
TABLE II

Operator ENERgy EFFiciency (X $10^{4}$ Mbits/Joule) With Respect to The FOT SCHEME FOR $z=0.1$

\begin{tabular}{|c||ccccc|ccccc|}
\hline \multicolumn{1}{|c||}{} & \multicolumn{5}{c|}{ Tolerant bidding } & \multicolumn{5}{c|}{ Non-tolerant bidding } \\
\hline \hline Algorithm & MNO $_{\mathbf{1}}$ & $\mathbf{M N O}_{\mathbf{2}}$ & $\mathbf{M N O}_{\mathbf{3}}$ & $\mathbf{M N O}_{\mathbf{4}}$ & $\mathbf{M N O}_{\mathbf{5}}$ & $\mathbf{M N O}_{\mathbf{1}}$ & $\mathbf{M N O}_{\mathbf{2}}$ & $\mathbf{M N O}_{\mathbf{3}}$ & $\mathbf{M N O}_{\mathbf{4}}$ & $\mathbf{M N O}_{\mathbf{5}}$ \\
\hline \hline MAS-L $=\mathbf{3}$ & $\infty$ & 3 & $\infty$ & $\infty$ & $\infty$ & $\infty$ & 3 & 2.2 & $\infty$ & $\infty$ \\
\hline ISO-L $=\mathbf{3}$ & $\infty$ & $\infty$ & 2.2 & 2.2 & 1 & $\infty$ & $\infty$ & 2.2 & 2.2 & 1 \\
\hline \hline MAS-L $=\mathbf{7}$ & $\infty$ & $\infty$ & $\infty$ & $\infty$ & $\infty$ & $\infty$ & 3 & $\infty$ & $\infty$ & $\infty$ \\
\hline ISO-L $=\mathbf{7}$ & $\infty$ & $\infty$ & 2.2 & 2.2 & 1 & $\infty$ & $\infty$ & 2.2 & 2.2 & 1 \\
\hline
\end{tabular}

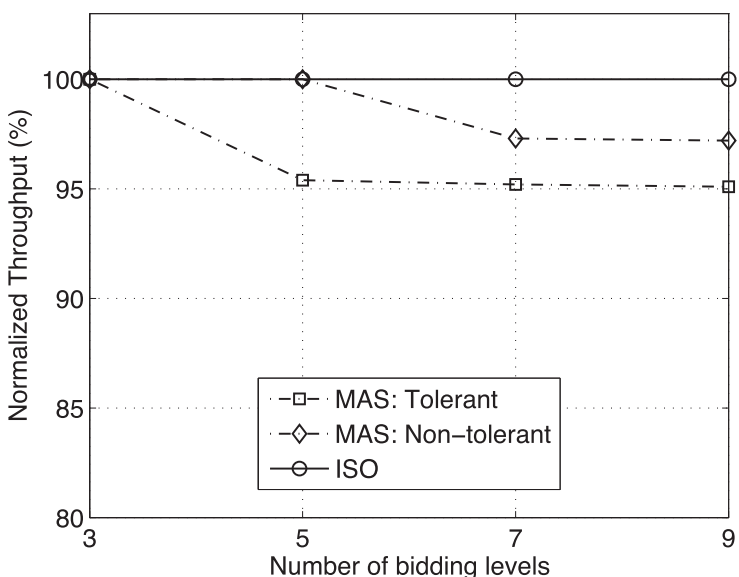

(a)

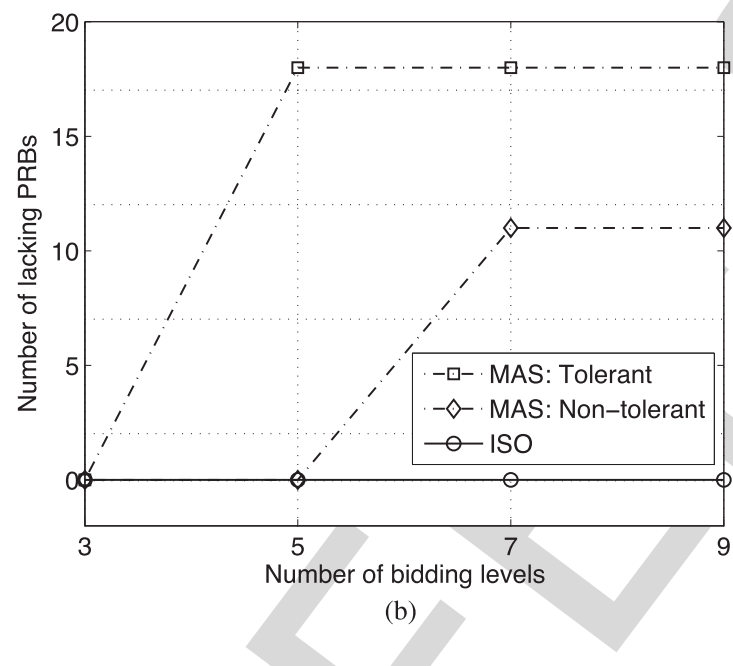

Fig. 9. (a) Normalized throughput for different bidding levels and strategies. (b) Number of lacking PRBs.

788 have their traffic served at zero energy cost, whereas the active 789 operators serve their own traffic without improving their situa790 tion. The proposed MAS eliminates this unfairness by offering 791 the chance to more MNOs to switch off their BSs, providing 792 them with extra incentives to participate in the auction. The 793 individual gains are remarkable of our proposal for both the 794 MNOs and the whole network.

795 Despite the importance of the energy efficiency results, the 796 deactivation of the BSs in the network and the traffic offloading 797 to the SCs potentially implies loss of connections. To that 798 end, we study the normalized throughput that represents the 799 percentage of served connections in the system. In Fig. 9(a), 800 it can be observed that, as the number of switched-off BSs 801 increases, the MAS approach experiences small losses (around
$5 \%$ and $3 \%$ for tolerant and nontolerant bidding, respectively). 802 The degraded performance is explained by the high number 803 of deactivated BSs, leading to the service of all the traffic 804 mainly by the SC network (since at most one BS remains 805 active). The MNOs are able to decide whether the throughput 806 performance can be sacrificed to achieve energy efficiency, or 807 even small losses are prohibitive (thus, a tolerant strategy is not 808 acceptable). The results in terms of lacking PRBs are given 809 in Fig. 9(b). As it is observed, a larger number of PRBs are 810 lacking, when the MAS strategy is applied. In our case, there 811 are different reasons for the degraded throughput performance. 812 The unserved users exist because either lower bids (thus, fewer 813 PRBs) are selected or the overall PRBs are not adequate for the 814 total offloaded traffic. However, the impact on the throughput is 815 negligible, as it was shown in Fig. 9(a).

816

2) Cost Metrics: The total annual network cost and the 817 individual annual gains for the operators and the third party, 818 compared with the state-of-the-art algorithm and having as a 819 benchmark the FOT scheme, are presented in Figs. 10 and 12, 820 respectively.

The annual network cost is given versus the different number 822 of bidding levels and for the tolerant [see Fig. 10(a)] and 823 nontolerant [see Fig. 10(b)] bidding strategies, respectively. 824 The total network cost is the sum of the average cost of all 825 MNOs and the third party for the operation of all infrastructures 826 (i.e., the active BSs and SCs). The first observation is that the 827 network cost is the same for level pairs $(l=3$ and $l=5)$ and 828 ( $l=7$ and $l=9$ ) for the MAS algorithm, due to the number 829 of switched-off BSs that is the same for the two cases. The 830 MAS scheme achieves a considerable reduction up to $94 \% 831$ and $96 \%$ for tolerant bidding, when compared with ISO and 832 FOT schemes, respectively. For the case of nontolerant bidding 833 strategy, the cost reduction is lower. This result is explained by 834 the fact that MNOs are more greedy, and in their attempt to 835 increase their economic gains, they do not offer high bids for 836 the requested capacity. As a consequence, the third party does 837 not accept the allocation of resources to low prices, and some 838 BSs may not be switched off. Concerning the ISO scheme, we 839 observe that the total annual cost is not affected by the different 840 bidding levels since, in ISO auction, only one bid is offered 841 based on the maximum traffic expectations, and fewer BSs are 842 deactivated. A clear comparison between MAS, for tolerant and 843 nontolerant bidding, and ISO is given in Fig. 11, where the 844 annual network cost behavior is highlighted.

The individual revenue of each MNO and the third party is 846 plotted in Fig. 12. We may observe that, similar to the energy 847 efficiency gains, the ISO scheme provides financial gains only 848 to particular operators $\left(\mathrm{MNO}_{1}\right.$ and $\left.\mathrm{MNO}_{2}\right)$ and particularly to 849 


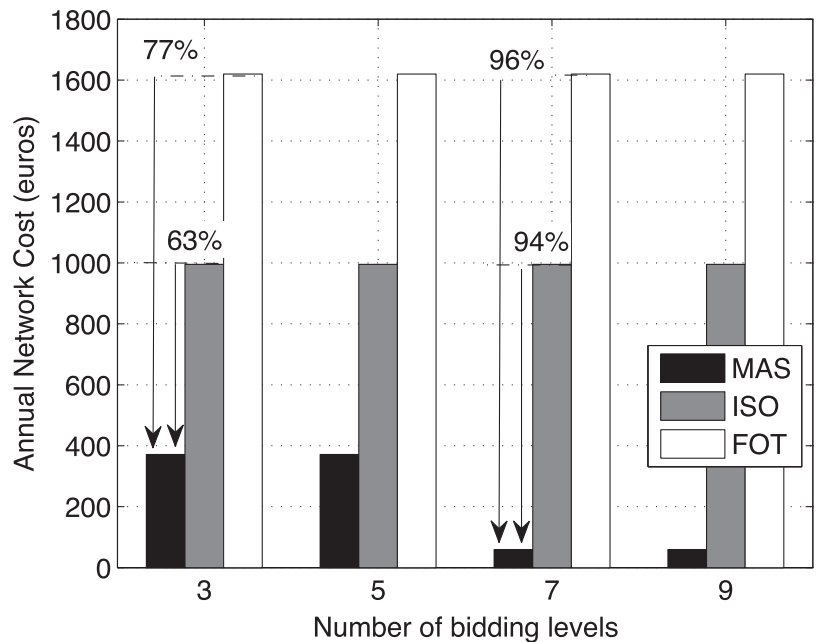

(a)

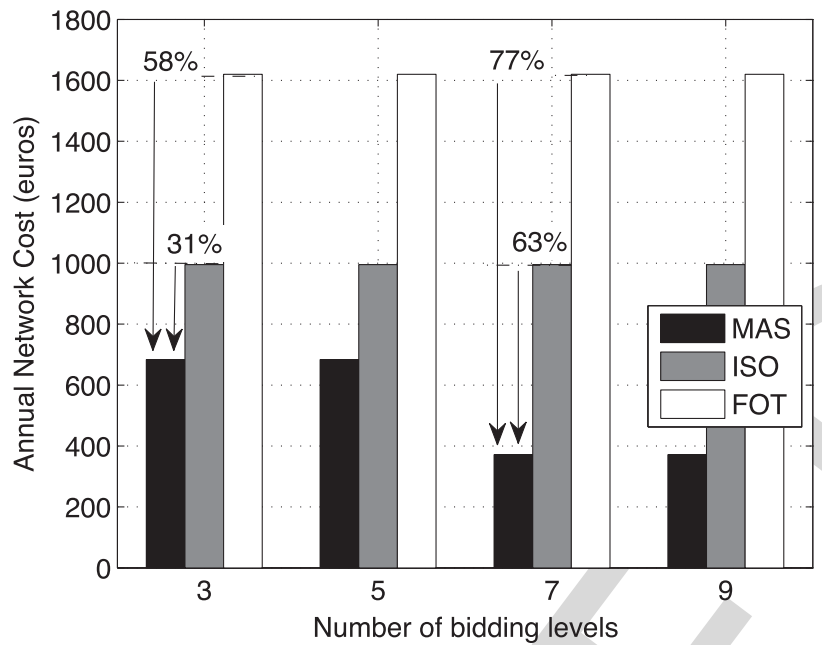

(b)

Fig. 10. Annual network cost for $z=0.1$ and different bidding strategies. (a) Tolerant bidding strategy. (b) Nontolerant bidding strategy.

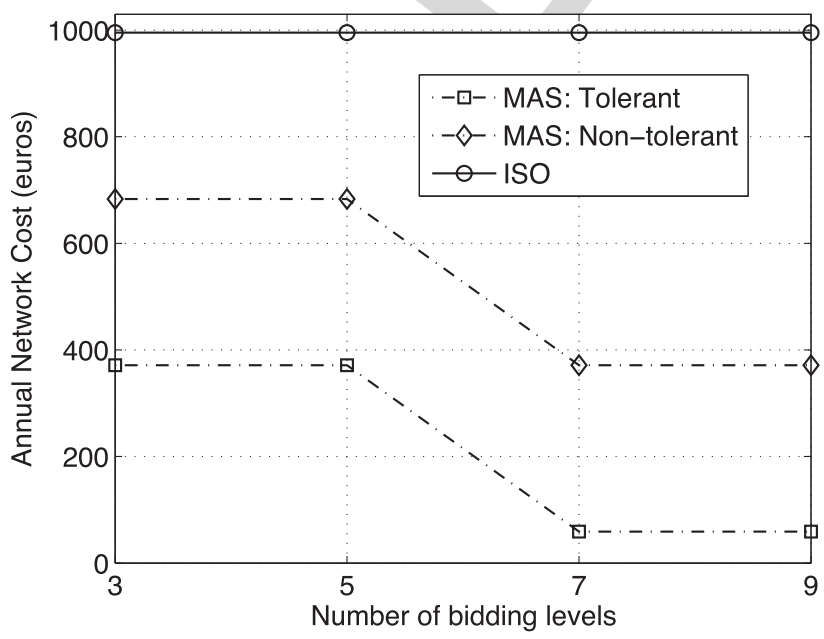

Fig. 11. Annual network cost for different bidding levels and strategies.

850 the MNOs that switch off their networks, whereas the economic 851 gains of the remaining operators are zero compared with the 852 FOT scheme since these MNOs keep their BSs active and serve

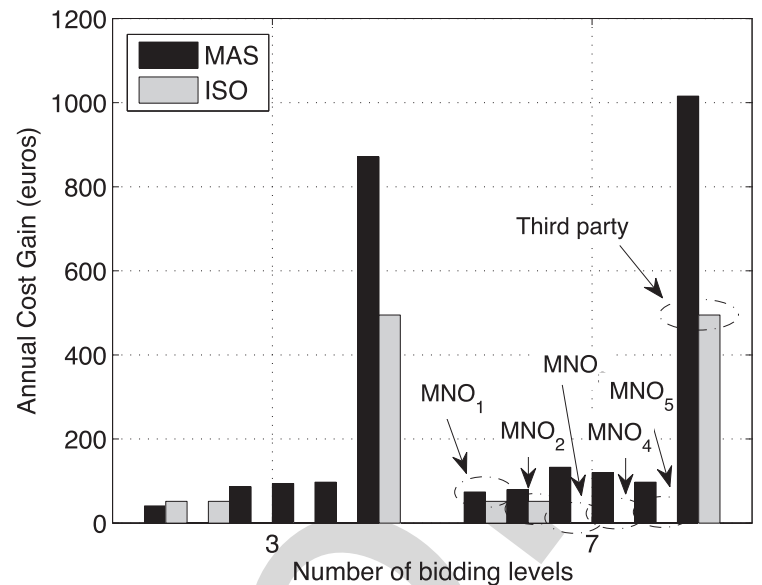

(a)

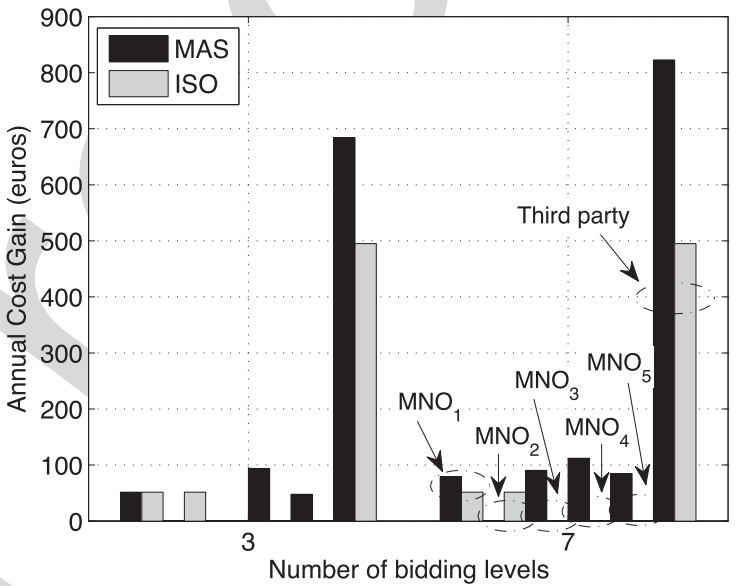

(b)

Fig. 12. Annual cost gain for $z=0.1$ and different bidding strategies. (a) Tolerant bidding strategy. (b) Nontolerant bidding strategy.

their own traffic without having any benefit from the auction. 853 On the other hand, for the proposed MAS approach, more 854 operators are able to have economic benefits, independently of 855 the particular number of levels. Furthermore, as the number of 856 bidding levels increases, the MAS algorithm achieves higher 857 financial gains for the operators due to the higher number 858 of combinations of bids offered. In addition, for the tolerant 859 bidding strategy [see Fig. 12(a)], the economic gains are higher 860 compared with the nontolerant behavior [see Fig. 12(b)].

\section{Discussion}

Based on the analysis in Sections IV-B and IV-C, we have 863 shown that the proposed MAS scheme outperforms the state-of- 864 the-art approaches in terms of energy efficiency (network and 865 individual), annual network cost, and individual cost gains. In 866 addition, it achieves balanced results for the MNOs with respect 867 to the individual energy efficiency and cost gains. Furthermore, 868 through a performance assessment, we have identified the sig- 869 nificance of the bidding behavior, the number of levels, and pa- 870 rameter $z$. In particular, better performance results are attained 871 in terms of energy efficiency, aggregate network cost, and indi- 872 vidual cost gains, when a higher number of levels are chosen 873 
874 and through tolerant bidding. Moreover, higher values of $z$ 875 achieve lower gains for both the MNOs and the third party. Fi876 nally, although minor losses may appear in terms of throughput, 877 the attained benefits with respect to energy efficiency, individ878 ual and network cost gains are remarkable and are adequate for 879 giving the necessary incentives to the MNOs and the third party 880 to apply the proposed auction-based switching-off strategy.

881

\section{CONCLUSION}

882 In this paper, motivated by the low BS utilization during 883 the night and the coexistence of multiple MNOs and third884 party SCs in the same area, we proposed a novel auction-based 885 offloading and switching-off algorithm that achieves energy 886 savings and cost reduction by encouraging MNOs to offload 887 their traffic and switch off the redundant BSs. Moreover, by 888 employing auction tools and novel bidding strategies, we intro889 duced a switching-off scheme that allows the MNOs to reduce 890 their expenditures. The proposed scheme has been evaluated 891 in terms of energy efficiency and cost metrics for various 892 conditions (different bidding levels and behaviors). The results 893 have shown that our proposal can significantly improve the 894 network energy efficiency, guaranteeing at the same time the 895 throughput. Regarding the financial costs/gains, the proposed 896 scheme provides higher cost benefits and fairness compared 897 with the state-of-the-art algorithms. It provides also interesting 898 insights concerning the rules and behaviors that the MNOs 899 should follow when they participate in an auction strategy. The 900 study of other schemes for the solution of the problem (such 901 as backwards induction) and the potential trade-offs would be 902 interesting research line for future works.

\section{REFERENCES}

[1] "Cisco visual networking index: Global mobile data traffic forecast update," Cisco, San Francisco, CA, USA, pp. 2014-2019, Feb. 2015.

2] X. Bao, Y. Lin, U. Lee, I. Rimac, and R. R. Choudhury, "Dataspotting: Exploiting naturally clustered mobile devices to offload cellular traffic," in Proc. IEEE INFOCOM, Apr. 2013, pp. 420-424.

[3] "Nokia networks: Solutions," Nokia, Espoo, Finland, 2015. [Online]. Available: http://networks.nokia.com/portfolio/solutions/heterogeneousnetworks

[4] L. Gao, G. Iosifidis, H. Jianwei, and L. Tassiulas, "Economics of mobile data offloading," in Proc. IEEE Workshop INFOCOM, Apr. 2013, pp. 3303-3308.

5] W. Dong et al. "iDEAL: Incentivized dynamic cellular offloading via auctions," in Proc. IEEE INFOCOM, Apr. 2013, pp. 755-763.

[6] S. Paris, F. Martignon, I. Filippini, and A. Capone, "A truthful auction for access point selection in heterogeneous mobile networks," in Proc. IEEE ICC, Jun. 2012, pp. 3200-3205.

[7] X. Zhuo, W. Gao, G. Cao, and S. Hua, "An incentive framework for cellular traffic offloading," IEEE Trans. Mobile Comput., vol. 13, no. 3, pp. 541-555, Mar. 2014.

[8] G. Fettweis and E. Zimmermann, "ICT energy consumption-trends and challenges," in Proc. WPMC, Sep. 2008, pp. 1-4.

[9] G. Auer et al., "How much energy is needed to run a wireless network?" IEEE Wireless Commun., vol. 18, no. 5, pp. 40-49, Oct. 2011.

[0] F. Han, Z. Safar, and K. J. R. Liu, "Energy-efficient base-station cooperative operation with guaranteed QoS," IEEE Trans. Commun., vol. 61, no. 8, pp. 3505-3517, Aug. 2013.

1] E. Oh, K. Son, and B. Krishnamachari, "Dynamic base station switchingon/off strategies for green cellular networks," IEEE Trans. Wireless Commun., vol. 12, no. 5, pp. 2126-2136, May 2013.

12] A. Bousia, E. Kartsakli, L. Alonso, and C. Verikoukis, "Dynamic energy efficient distance-aware base station switch on/off scheme for LTEAdvanced," in Proc. IEEE GLOBECOM, Dec. 2012, pp. 1532-1537.
[13] A. Bousia, E. Kartsakli, A. Antonopoulos, L. Alonso, and C. Verik- 936 oukis, "Game theoretic infrastructure sharing in multi-operator cellular 937 networks," IEEE Trans. Veh. Technol., DOI: 10.1109/TVT.2015.2445837, 938 to be published.

[14] J. Bartelt, A. Fehske, H. Klessig, G. Fettweis, and J. Voigt, "Joint band- 940 width allocation and small cell switching in heterogeneous networks," 941 in Proc. IEEE VTC-Fall, Sep. 2013, pp. 1-5.

942

[15] Y. S. Soh, T. Q. S. Quek, M. Kountouris, and H. Shin, "Energy efficient 943 heterogeneous cellular networks," IEEE J. Sel. Areas Commun., vol. 31, 944 no. 5, pp. 840-850, May 2013.

[16] M. H. Alsharif, R. Nordin, and M. Ismail, "A review on intelligent base 946 stations cooperation management techniques for greener LTE cellular 947 networks," J. Commun., vol. 9, no. 12, pp. 937-945, Dec. 2014.

[17] S. Paris, F. Martignon, I. Filippini, and L. Chen, "A bandwidth trad- 949 ing marketplace for mobile data offloading," in Proc. IEEE INFOCOM, 950 Apr. 2013, pp. 430-434.

[18] M. A. Marsan and M. Meo, "Network sharing and its energy bene- 952 fits: A study of European mobile network operators," in Proc. IEEE 953 GLOBECOM, Dec. 2013, pp. 2561-2567.

[19] "Small cell market status," Small Cell Forum, U.K., Feb. 2013. [Online]. 955 Available: http://www.smallcellforum.org/resources-reports 956

[20] "3rd generation partnership project; technical specification group services 957 and system aspects; telecommunication management; study on Energy 958 Savings Management (ESM) (Release 10)," Third-Generation Partnership 959 Project, Sophia Antipolis Cedex, France, Tech. Rep., 3GPP TR 32.826, 960 V10.0.0, Mar. 2010.

[21] "Mobile traffic forecasts 2010-2020 Report," UMTS Forum, London, 962 U.K., Jan. 2011.

[22] V. Krishna, Auction Theory. San Diego, CA, USA: Academic, 2010. 964

[23] A. Bousia, E. Kartsakli, A. Antonopoulos, L. Alonso, and C. Verikoukis, 965 "Sharing the small cells for energy efficient networking: How much does 966 it cost?" in Proc. IEEE GLOBECOM, Dec. 2014, pp. 2649-2654. 967

[24] Y. Sawaragi, I. Hirotaka, and T. Tanino, Theory of Multiobjective Opti- 968 mization, 1st ed. New York, NY, USA: Academic, 1985.

[25] T. Weise, Global Optimization Algorithms-Theory and Application, 2nd 970 ed. Hefei, China: Thomas Weise, 2009. [Online]. Available: http://www. 971 it-weise.de/

[26] R. L. Carraway, T. L. Morin, and H. Moskowitz, "Generalized dynamic 973 programming for multicriteria optimization," Eur. J. Oper. Res., vol. 44, 974 no. 1, pp. 95-104, Jan. 1990.

[27] H. G. Beyer and K. Deb, "On self-adaptive features in real-parameter 976 evolutionary algorithms," IEEE Trans. Evol. Comput., vol. 5, no. 3, 977 pp. 250-270, Jun. 2001.

[28] C. A. Coello, G. B. Lamont, and D. A. Van Veldhuizen, Evolutionary Al- 979 gorithms for Solving Multi-Objective Problems, ser. Genetic and Evolu- 980 tionary Computation, 2nd ed. Berlin, Germany: Springer-Verlag, 2007. 981

[29] L. M. Schmitt, "Theory of genetic algorithms II: Models for genetic oper- 982 ators over the string-tensor representation of populations and convergence 983 to global optima for arbitrary fitness function under scaling," Theoretical 984 Comput. Sci., vol. 310, pp. 181-231, 2004.

[30] "Ericsson mobility report: On the pulse of the networked society," 986 Ericsson, Stockholm, Sweden, Tech. Rep. Nov. 2014.

[31] "Sitefinder: Mobile Phone Base Station Database," Ofcom, London, 988 U.K., Mar. 2015. [Online]. Available: http://sitefinder.ofcom.org.uk/search 989

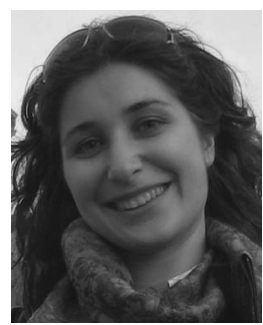

Alexandra Bousia (M'15) received the B.S. and 990 M.S. degrees from the University of Thessaly, Volos, 991 Greece, in 2008 and 2009, respectively. She is cur- 992 rently working toward the $\mathrm{Ph} . \mathrm{D}$. degree in wireless 993 communications with the Department of Signal The- 994 ory and Communications, Universitat Politècnica de 995 Catalunya (UPC), Barcelona, Spain. 996

She is also currently a Marie Curie Researcher 997 with UPC. Since 2011, she has been involved in 998 several national and European projects. Her research 999 interests include wireless networks, medium-access- 1000 control protocols, energy-efficient protocols, and radio resource management 1001 algorithms.

Ms. Bousia received the Best Paper Award at the IEEE Global Communica- 1003 tions Conference in 2014. 


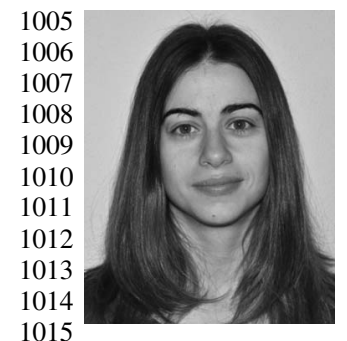

Elli Kartsakli (SM'15) received the Bachelor's degree in electrical and computer engineering from the National Technical University of Athens, Athens, Greece, in 2003; the M.Sc. degree in mobile and satellite communications from the University of Surrey, Surrey, U.K., in 2004; and the Ph.D. degree in wireless telecommunications from the Universitat Politècnica de Catalunya (UPC), Barcelona, Spain, in 2012.

She is currently a Postdoctoral Researcher with UPC. She has participated in several national and 1016 European projects (e.g., GREENET and WSN4QoL). Her main research inter1017 ests include wireless networking, channel access protocols, and energy-efficient 1018 protocols.

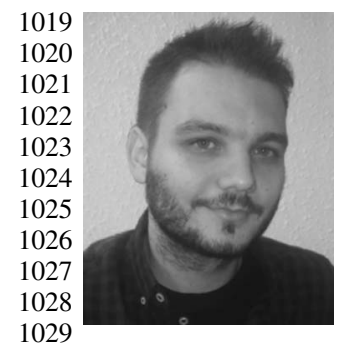

Angelos Antonopoulos (SM'15) received the Ph.D. degree from the Universitat Politècnica de Catalunya, Barcelona, Spain, in 2012.

$\mathrm{He}$ is currently with the Telecommunications Technological Center of Catalonia, Barcelona, Spain. $\mathrm{He}$ has participated in several European projects. He is the author of more than 50 papers on various topics, including cooperative communications, network coding, and green networking.

Dr. Antonopoulos has received an award from the Technical Chamber of Greece for Exceptional Grad1030 uate Performance. In 2014, he also received the Best Paper Award at the IEEE 1031 Global Communications Conference and the Best Demo Award at the IEEE 1032 International Workshop on Computer-Aided Modeling Analysis and Design of 1033 Communication Links and Networks. In January 2015, he was nominated as 1034 Exemplary Reviewer for the IEEE COMMUNICATIONS LETTERS.

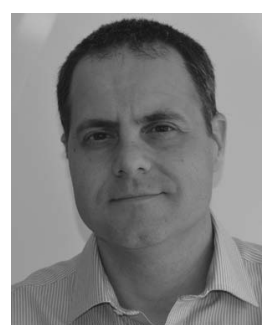

Luis Alonso (SM'XX) received the Ph.D. de- 1035 AQ6 gree from the Universitat Politècnica de Catalunya, 1036 Barcelona, Spain, in 2001.

Since 2006, he has been an Associate Professor 1038 with UPC. He is a Cofounder of the Wireless Com- 1039 munications and Technologies Research Group 1040 (WiComTec). He is the author of 40 research papers, 1041 one book, 12 book chapters, and more than 100 papers 1042 in international congresses. His research interests 1043 include medium access protocols, cross-layer opti- 1044 mization, cooperative transmissions, cognitive radio, 1045 and quality of service for all kinds of wireless networks. 1046 Dr. Alonso has received several best paper awards.

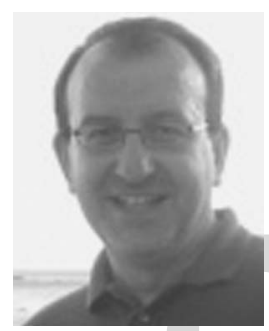

Christos Verikoukis (SM'07) received the Ph.D. de- 1048 gree from the Universitat Politècnica de Catalunya, 1049 Barcelona, Spain, in 2000.

1050

$\mathrm{He}$ is currently a Senior Researcher with the Tele- 1051 communications Technological Center of Catalo- 1052 nia, Barcelona, and an Adjunct Professor with 1053 the University of Barcelona. He is the author or 1054 coauthor of 86 journal papers, over 160 conference 1055 papers, three books, 14 book chapters, and two 1056 patents. He has participated in more than $30 \mathrm{com}-1057$ petitive projects and has served as a principal in- 1058 vestigator of national projects. He has supervised $15 \mathrm{Ph} . \mathrm{D}$. students and five 1059 postdoctoral researchers.

1060

Dr. Verikoukis currently serves as the Chair of the IEEE Communication 1061 Society Communications Systems Integration and Modeling Technical Com- 1062 mittee. He received the Best Paper Award at the IEEE International Conference 1063 on Communications in 2011 and the IEEE Global Communications Conference 1064 in 2014 and 2015, as well as the EURASIP 2013 Best Paper Award for the 1065 Journal on Advances in Signal Processing. 


\section{AUTHOR QUERIES}

\section{AUTHOR PLEASE ANSWER ALL QUERIES}

AQ1 = Please check if the expanded form provide for "PRBs" is appropriate. If not, kindly provide the necessary correction.

AQ2 = If possible, please provide an updated image of Fig. 5 so that the labels for the $\mathrm{x}$ - and $\mathrm{y}$-axis are enlarged for improved readability.

AQ3 = Please expand the ILP acronym.

AQ4 = Please update the images of Figs. 6(a) and (b) so that the x-axis label is amended to reflect "Thirdparty..." instead of "Third party..."

AQ5 = Please provide publication update in Ref. [13].

AQ6 = Please provide membership history of author "Luis Alonso."

END OF ALL QUERIES 